This item was submitted to Loughborough's Research Repository by the author.

Items in Figshare are protected by copyright, with all rights reserved, unless otherwise indicated.

\title{
Simulation of receptivity and induced transition from discrete roughness elements
}

PLEASE CITE THE PUBLISHED VERSION

http://dx.doi.org/10.1007/s10494-015-9636-y

\section{PUBLISHER}

(C) Springer Science+Business Media

\section{VERSION}

AM (Accepted Manuscript)

\section{PUBLISHER STATEMENT}

This work is made available according to the conditions of the Creative Commons Attribution-NonCommercialNoDerivatives 4.0 International (CC BY-NC-ND 4.0) licence. Full details of this licence are available at: https://creativecommons.org/licenses/by-nc-nd/4.0/

\section{LICENCE}

CC BY-NC-ND 4.0

\section{REPOSITORY RECORD}

Mistry, Vinan I., Gary Page, and Jim McGuirk. 2015. "Simulation of Receptivity and Induced Transition from Discrete Roughness Elements”. Loughborough University. https://hdl.handle.net/2134/18971. 


\title{
Simulation of Receptivity and Induced Transition
}

\section{From Discrete Roughness Elements}

\author{
V. I. Mistry, G. J. Page, J. J. McGuirk \\ Dept. of Aero. \& Auto. Eng., Loughborough University, Loughborough LE11 3TU, UK. \\ +44 1509227211 \\ +44 1509227241 \\ j.j.mcguirk@lboro.ac.uk
}

\begin{abstract}
Simulations have been carried out to predict the receptivity and growth of crossflow vortices created by Discrete Roughness Elements (DREs). The final transition to turbulence has also been examined, including the effect of DRE spacing and freestream turbulence. Measurements by Hunt and Saric [15] of perturbation mode shape at various locations were used to validate the code, in particular for the receptivity region. The WALE sub-grid stress (SGS) model was adopted for application to transitional flows, since it allows the SGS viscosity to vanish in laminar regions and in the innermost region of the boundary layer when transition begins. Simulations were carried out for two spanwise wavelengths: $\lambda=12 \mathrm{~mm}$ (critical) and $\lambda=6 \mathrm{~mm}$ (control), and for roughness heights (k) from $12 \mu \mathrm{m}$ to $42 \mu \mathrm{m}$. The base flow considered was an ASU (67)-0315 aerofoil with $45^{\circ}$ sweep at $-2.9^{0}$ incidence and with onset flow at a chordbased Reynolds number $\operatorname{Re}_{\mathrm{c}}=2.4 \times 10^{6}$. For $\lambda=12 \mathrm{~mm}$ results showed, in accord with the experimental data, that the disturbance amplitude growth rate was linear for $k=12 \mu \mathrm{m}$ and $24 \mu \mathrm{m}$, but the growth rate was decreased for $\mathrm{k}=36 \mu \mathrm{m}$. Receptivity to $\lambda=6 \mathrm{~mm}$ roughness showed equally good agreement with experiments, indicating that this mode disappeared after a short distance to be replaced by a critical wavelength mode. Analysis of the development of modal disturbance amplitudes with downstream distance showed regions of linear, non-linear, saturation, and secondary instability behaviour. Examination of breakdown to turbulence revealed two possible routes: the first was 2D-like transition (probably Tollmien-Schlichting waves even in the presence of crossflow vortices) when transition occurred beyond the pressure minimum; the second was a classical crossflow vortex secondary instability, leading to the formation of a turbulent wedge.
\end{abstract}

Keywords Large Eddy Simulation, Discrete Roughness Elements, Receptivity, Transition

\section{Introduction}

Continuing legislative pressure to reduce carbon emissions and environmental impact from civil aviation operations (e.g. Flightpath 2050 [1]) has driven much research into aerodynamic drag reduction to reduce fuel burn. For a transport aircraft in cruise, the majority of drag is due to skin friction from turbulent boundary layers. Arnal and Archambaud [2] estimate that extended retention of laminar flow on transport aircraft wings could reduce drag by $15 \%$, with associated fuel and emissions savings. The introduction of control methods to achieve extended laminar flow thus has significant potential for reduced skin friction drag, see Green [3]. Moving the onset of laminar/turbulent transition as far aft as 
possible can only be achieved via combined manipulation of all mechanisms which trigger transition. For swept-wings, judicious design of attachment line Reynolds number for a given leading edge sweep and radius is successful at delaying attachment line transition (Poll [4]). Similarly, aerofoil shaping - to create favourable pressure gradients and to minimise the pressure recovery region - is known to supress the Tollmien-Schlichting waves that lead to mid-chord transition. For highly swept-wings with strong favourable pressure gradients, a third mechanism known as crossflow transition becomes important. The primary instability is in this case associated with the presence of an inflection point in the crossflow velocity boundary layer profile and is a further source of inviscid instability and transition (Saric et al. [5]). This mechanism often causes transition close to the leading edge. Whilst linear stability theory indicates unstable (amplified) modes of both stationary and travelling types occur, excited initially by minute surface roughness features, experimental evidence (Saric et al. [5]) suggests that at the low freestream turbulence levels relevant to flight, it is the stationary mode that dominates.

Crossflow transition is the transition process of relevance to the present work. Naturally-occurring surface roughness is an important initiator; Radeztsky et al. [6] conducted experiments which demonstrated how a painted model ( $9 \mu \mathrm{m}$ rms roughness) induced transition $30 \%$ chord earlier than with a polished model $(0.25 \mu \mathrm{m}$ rms roughness). White and Saric [7] have provided a concise description of the overall transition process. This requires first and foremost a thorough understanding of the receptivity of the laminar boundary layer to the flow disturbance initiated by surface roughness, since this provides the initial conditions for primary instability growth. The primary disturbance created takes the form of arrays of vortices aligned closely with the streamwise flow. These are amplified as they develop downstream, displaying linear and non-linear growth until their amplitude saturates at $\sim 20 \%$ of the freestream velocity. The nature of the flow distortion created (more details given below) then allows unstable secondary instabilities to appear; these are subsequently observed to breakdown very rapidly into turbulence. The distinguishing feature of crossflow transition, identified early on from flow visualisation studies, was the appearance of surface streaks with a distinctive spanwise spacing, leading to a distinctive 'sawtooth' shape to the laminar/turbulent transition, cause by a breakdown into turbulence with a particular 'turbulentwedge-shaped' signature (see Fig. 1, from Dagenhart and Saric [8]).

The interest in crossflow vortices excited by surface roughness led to an experimental study by Saric et al. [9], who introduced a row of roughness elements on the swept-wing surface near to the leading edge with a spanwise wavelength chosen to coincide with the most unstable stationary disturbance of the laminar boundary layer profile identified from linear stability analysis. It was observed that the transition location then moved forward considerably. A second observation was that, in addition to the primary instability, only super-harmonics of the primary wavelength were excited in the downstream flow development, no sub-harmonics appeared. These observations encouraged Saric et al. [9] to propose and demonstrate a method for controlling (delaying) crossflow vortex induced transition. This concept involved the use of a row of artificially introduced Discrete Roughness Elements (DREs) at a spanwise wavelength chosen to excite a specific mode (control mode) whose spanwise wavelength was shorter than that of the most unstable primary disturbance (critical mode). The idea here is that the shorter wavelength control 
mode modifies the base state of the boundary layer in such a way that, for an initial region, the control mode grows preferentially and suppresses growth of the more unstable longer critical wavelength mode, which no longer grows as it would in the absence of the control mode. The amplitude of the control wavelength eventually saturates and decays downstream, to be replaced by the naturally unstable critical wavelength, but the delayed growth of this then takes longer to trigger transition, and extended laminar flow is possible. Saric et al. [9] demonstrated a delay in transition to turbulence of $\sim 15 \% c$ with this method ( $c$ is the swept-wing chord). However, in flight test measurements - designed to test the wind tunnel results of Saric et al. [9] at higher Reynolds numbers - Carpenter et al. [10] noted that when the control mode was excited it had no effect on transition location unless above a critical roughness height. Contradictory indications occurred in other experiments; for example the wind tunnel data of Radeztsky et al. [6] showed that different roughness heights advanced the transition location upstream as long as amplitude saturation was not reached, but the opposite was concluded by Reibert et al. [11] - one explanation for this is that [11] used isolated roughness elements rather than the spanwise array used in [10]. Similarly, some experimental studies indicate an initial linear growth rate of the flow perturbation, for example Borodoulin et al. [12], whilst others have observed a quadratic relationship in the 'linear' region (Kurian et al. [13]). Note that a key difference between these experiments is that one used a swept-wing while the other used a swept flat plate. The response to surface roughness is somewhat different for boundary layers developing in these flows; curvature, for example, is known to have a stabilising effect on stationary mode growth, and the relative positions of the crossflow neutral stability points are also different. There are clearly unanswered questions in connection with the response of swept-wing laminar boundary layers to the initial disturbance amplitude.

The above discrepancies prompted the experiments of Hunt [14] (see also Hunt and Saric [15]), who reported a wind tunnel study of the receptivity process, with particular reference to initial disturbance amplitude for DREs characterised by a row of cylinders. Hot-wire measurements were conducted to obtain information on the effect of variations in initial disturbance amplitude (DRE height) over a range of wing chord Reynolds numbers. A single row of (uniform shape) DREs at various spatial wavelengths and with increasing DRE height were introduced on a constant chord wing at $45^{\circ}$ sweep. It is important to note that this investigation was carried out in a modified version of the wind tunnel used in the measurements of Saric et al. [9] (Note - [9] and [14] used different test models although with the same chord length and sweep angle). The data from [14], [15] indicated transition in the baseline swept-wing flow (i.e. without any added artificial roughness) to be as far aft as $80 \%$ chord. This was somewhat surprising since this was significantly further aft than previously measured $\left((\mathrm{x} / \mathrm{c})_{\mathrm{tr}}\right.$ was $\sim 65 \%$ in [9]). In addition, transition at $80 \%$ chord was downstream of the pressure minimum location and in a region of steep adverse pressure gradient; the observed transition line was quite uniform spanwise, which was interpreted by the authors as due to transition being caused by a Tollmien-Schlichting instability in the decelerating boundary layer region. One possible cause of the change in baseline transition behaviour between the two wind tunnel studies suggested by the authors was a reduction in freestream turbulence level achieved by wind tunnel 
modifications carried out when moving the wind tunnel between the two University laboratories - the freestream turbulence intensity had been reduced from $0.04 \%$ in [9] to $0.02 \%$ in [15]. Further, the transition location was noted in [15] to be sensitive to DRE height when DREs at the critical wavelength were introduced, contrary to the observations of Reibert et al. [11]. Finally, tests with DREs spaced at a control wavelength in [14] indicated this caused the transition to move forward rather than aft as would be expected if control was effective. It was suggested that with the large extent of laminar flow in the baseline case (past the pressure minimum), DRE disturbance amplitudes may have been too large for control purposes, or again perhaps the change in freestream turbulence level had altered the mode of transition from being dominated by a stationary disturbance to being driven by travelling waves (which are known to be more sensitive to freestream turbulence than stationary vortices). This issue of a transition being downstream of the pressure minimum even in the baseline (no DRE) flow needs careful consideration. Several authors have commented upon this: (i) Carrillo [16] has reported "...transition location aft of the pressure minimum is likely due to Tollmien-Schlichting waves rather than crossflow vortices"; (ii) Saric et al. [17] noted "...as the unmodified transition location is relatively far aft (in the vicinity of the pressure minimum) the potential for transition control is minimal; (iii) and finally, to prevent this issue making interpretation of control effectiveness difficult, Lovig et al. [18] in their experiments reported: ".....for some tests the baseline $(x / c)_{\text {tr }}$ was too far downstream to demonstrate transition control" and took the step of adding artificial surface roughness in the form of a $70 \mu \mathrm{m}$ rms rough polyamide tape attached at the model leading edge to destabilise the boundary layer and hence move transition forward in their baseline (no DRE) case (from $80 \%$ to $45 \%$ chord) to create potential for transition delay. This problem will clearly affect both measurement and computational investigations of transition, but seems to have had little consideration in the latter to date, but should also occur in the present simulations if these are to reflect the experimental conditions of Hunt and Saric [15] appropriately.

To provide better understanding of the effects of freestream turbulence, Lovig et al. [18] and Downs and White [19] have carried out recent experimental programmes. It is generally accepted that the flight environment corresponds to low levels of turbulence (intensity less than $0.05 \%$ according to Riedel and Sitzmann [20] and Reshotko et al. [21]) and variations in the range $0.016 \%$ (low), $0.054 \%$ (intermediate) to $0.19 \%$ (high) were examined in [19]. It was shown in [19] that increased freestream turbulence had little effect on the receptivity; initiation of the stationary mode was observed to be dominated by surface roughness rather than freestream turbulence. For traveling modes, freestream turbulence plays the dominant role, setting the initial conditions. In terms of streamwise mode development, freestream turbulence acted to attenuate the amplitude saturation level, and decay was even seen at the highest intensity level (0.19\%). Again freestream turbulence increase had the expected opposite effect on traveling waves, increasing their growth rate. Similar observations were made when critical DREs were studied, but the control wavelength responded much less to increases in freestream turbulence intensity. In both baseline and critical DRE cases, increasing freestream turbulence advanced the transition location by $10 \% c$, but had little overall effect for the control wavelength (which delayed transition by $20 \%$ c relative to the critical case under all conditions). It was argued that for the control disturbance, rather than helping to 
destabilise the boundary layer, when exposed to elevated levels of freestream turbulence, growth of the travelling wave crossflow mode was excited and became the dominant transition path. In the investigation into freestream turbulence effects reported in [18], after the modification to ensure baseline transition was forward of the pressure minimum, the addition of a control roughness DRE row was very effective, moving transition from $x / c=45 \%$ to $x / c=75 \%$ as roughness height increased from $11 \mu \mathrm{m}$ to $66 \mu \mathrm{m}$. These results indicate that the effect of the combination of freestream turbulence and roughness on crossflowinduced transition is complex and requires further work to establish improved understanding.

The final stage of the transition process is the appearance of secondary instabilities, which have recently been studied experimentally by White and Saric [7] and computationally by Malik et al. [22] and Li et al. [23]. The latter have used a combination of non-linear parabolised stability equations (NLPSES) and a linear planar pde-based eigenvalue analysis. The onset of the secondary instability phase occurs when the primary vortex has saturated and the distorted mean flow displays strong inflectional velocity profiles; these are unstable and secondary instabilities of two types occur. These were labelled y-modes and zmodes, when successfully predicted in [23], and have subsequently been measured in detail in [7]; the terminology is related to whether the instability is driven by the inflectional profile in the wall-normal ( $y$ mode) or spanwise (z-mode) direction. The measurements of [22] revealed distinctive signatures of these modes, and locations which can be used to identify these, the $y$-modes being localised on the top of the primary vortex and the z-mode wrapped into its trailing side shear layer. These observations are an important source of validation data for computational approaches that are aimed at predicting transition paths.

In terms of predictive approaches for crossflow transition, as noted above, the most successful theoretical approach is based on NPSEs (Haynes and Reed [24]). This has provided results that indicate good agreement with experiments, matching both growth rate and saturation amplitude of the primary instability. However, the NPSE method, whilst indicating the transition location, cannot model the subsequent breakdown into turbulence. RANS CFD has not indicated much success at modelling of transitional flows, although the recent approach developed by Menter et al. [25] has shown some promise. This uses a k- $\omega$ SST model, additional transport equations for intermittency, and a locally-calculated vorticity Reynolds number in order to provide a link to empirical correlations based on momentum thickness Reynolds number. The approach has proven successful for natural transition in turbomachinery and wind-turbine applications, but unfortunately, no similar robust empirical correlations for crossflow transition are currently available.

Various authors have used Direct Numerical Simulation (DNS) to study DRE-induced crossflow transition. Wassermann and Kloker [26], [27] considered a swept flat plate and observed the streaky nature of the crossflow disturbance. At the critical wavelength this caused substantial mean flow distortion, however, when shorter (control) wavelengths were introduced, strong regions of deceleration were observed, the mean flow distortion weakened the growth of all other modes and the regions of local deceleration observed for the critical wavelength were weaker in amplitude. Hosseini et al. [28] introduced artificial unsteady background disturbances (localised pulsed volume forces) to simulate DREs 
at a control wavelength as well as natural roughness; transition was predicted to move downstream using the control disturbance. The most recent DNS study, by Kurz and Kloker [29], has argued that, in general, the peak magnitude of the most amplified stationary crossflow instability scales superlinearly with DRE height, with true linear behaviour only being found for DRE shapes with a zero spanwise mean (alternating bumps and dimples of similar shape); they suggest that superlinearity is related to the changing aspect ratio of DREs of fixed diameter as height is increased.

Use of specialist DNS codes as just described is clearly the best route to improve fundamental understanding of crossflow-induced transition. However, in order to achieve the resolution required at higher Reynolds numbers, DNS studies often involve some form of embedding of the DNS domain inside larger domains where lower fidelity numerical solutions are provided as boundary conditions to the DNS zone. Even in such cases, the freestream Reynolds numbers of DNS studies are inevitably restricted. For industry the extent that Large Eddy Simulation (LES) codes - suitable for practical engineering applications characterised by high Reynolds number - are able to capture the various stages of receptivity, primary/secondary instabilities, and breakdown into turbulence is an important issue. This will require careful attention to be paid to the sub-grid scale (SGS) model, since initial (unsteady) laminar flow behaviour must not be contaminated by added 'viscosity', and the same is true of the laminar and buffer regions of turbulent boundary layers after transition. References in the literature describing work that has attempted to study the performance of LES CFD for crossflow transition are rare. Schlatter [30] applied LES to predict natural transition in channel flows, showing good agreement with DNS at a fraction of the cost. Similarly, Sayadi and Moin [31] applied LES to natural transition in a zero pressure gradient boundary layer. Various SGS models were tried, with the dynamic version of the Smagorinsky model (which forces the SGS viscosity to zero in the early transition region) being successful at capturing transition location, although over-predicting the skin friction after breakdown to turbulence. The only direct application to crossflow vortices was the work of Huai et al. [32], where LES was applied to a swept boundary layer on a flat plate. Both stationary and travelling crossflow vortex disturbances were input using steady and randomamplitude suction and blowing. The evolution of the input vortices was observed to display linear growth, followed by saturation due to vortex 'roll-over', and double inflectional velocity profiles. Whilst this work appears to show encouraging results, the work was conducted at a low chord-based Reynolds number, with the base flow taken from the DLR Göttingen Prinzip experiment (see [33]). For this case it has been shown by Bonfigli et al. [33] using high-order DNS that the crossflow in the turbulent boundary layer is too low to influence the turbulent main flow profile, so it is contradictory that the LES results of [32] indicated any influence. The appearance of super-harmonics and the absence of sub-harmonics were also noted. In addition, no validation against experimental data was attempted, and the simulation was not continued through to where full transition to turbulence had taken place.

Based on the above review, it is clear that there are many questions still unanswered concerning predictive methods for crossflow-induced transition. This is particularly true of the capabilities of LES CFD for this problem. The aim of the present work is to examine the predictive capability of LES for all aspects of the crossflow-induced process: initial receptivity, linear and non-linear growth and saturation, 
secondary instability, and final breakdown into turbulence. The experimental configuration used by Hunt and Saric [15] has been selected as the test case for the present work, since this provides detailed experimental data of the all-important first receptivity phase and allows aspects such as critical and control DRE disturbance development to be explored and validated. The issue of baseline (no DREs) transition location aft of the pressure minimum was experienced in this data set and if this occurs in the simulation possible reasons for this can be assessed. The effect of freestream turbulence level will also be explored. The approach to this will initially be to carry out simulations with and without DREs with no turbulence in the freestream. This is not because these are relevant conditions for a flight environment - they are clearly not; rather these represent benchmark results against which to compare behaviour when freestream turbulence is introduced whilst keeping all other conditions the same. It is important to note that in all simulations no attempt has been made to take account of naturally-occurring surface roughness; the swept-wing wall is treated throughout as a fluid-mechanically smooth surface. This means that any predictions of transition location cannot be compared in an absolute sense with measurements since the initiation process for the primary instability will be different in simulations (essentially prompted by roundoff error) and measurement (real surface roughness). However, the trends predicted when critical or control DREs are introduced or freestream turbulence is added are of interest when compared to the trend seen in measurements, and this is the primary objective of the present study.

\section{Computational Method}

\subsection{LES governing equations}

Conventional low-pass spatially-filtered forms of the Navier-Stokes equations are used in the current LES formulation; an overbar indicates a large scale (or resolved) component, whereas a prime indicates a sub-grid scale (or residual) component. An incompressible, isothermal version of these equations is used (no energy equation required) since low Mach No. and constant density assumptions are valid for the experiments considered. The basic 'top-hat' filter operation (see Sagaut [34]) was adopted and the governing equations are written in Cartesian tensor format:

$$
\begin{gathered}
\frac{\partial \bar{u}_{i}}{\partial x_{i}}=0 \\
\frac{\partial \bar{u}_{i}}{\partial t}+\frac{\partial \bar{u}_{i} \bar{u}_{j}}{\partial x_{j}}=-\frac{1}{\rho} \frac{\partial \bar{p}}{\partial x_{i}}+\frac{\partial}{\partial x_{j}}\left[v\left(\frac{\partial \bar{u}_{i}}{\partial x_{j}}+\frac{\partial \bar{u}_{j}}{\partial x_{i}}\right)\right]-\frac{\partial \tau_{i j}}{\partial x_{j}} \\
\tau_{i j}=\left(\overline{u_{i} u_{j}}-\bar{u}_{i} \bar{u}_{j}\right)
\end{gathered}
$$

$\tau_{\mathrm{ij}}$ are the residual scale stresses and to close these equations an SGS model is required.

\subsection{SGS model}


The success of LES for transitional flows is highly dependent on the SGS model. For transitional flows, a suitable SGS model must deal with laminar, transitional, as well as fully turbulent regions. The model should leave laminar flow unaffected and only become effective when non-linear interactions generate eddies smaller than the mesh size. The present aim is to enable high-fidelity modelling at lower computational expense than DNS; the SGS model must thus introduce minimal computational cost. Most SGS models are based on an eddy-viscosity assumption; the most common example being the Smagorinsky [35] model:

$$
\begin{gathered}
\tau_{i j}=-2 v_{s g s} \bar{S}_{i j}+\frac{1}{3} \tau_{k k} \delta_{i j} \quad \bar{S}_{i j}=\frac{1}{2}\left(\frac{\partial \bar{u}_{i}}{\partial x_{j}}+\frac{\partial \bar{u}_{j}}{\partial x_{i}}\right) \\
v_{s g s}=\left(C_{S} \Delta^{2}\right)|\bar{S}| \quad \bar{S}=\sqrt{2 \bar{S}_{i j} \bar{S}_{i j}}
\end{gathered}
$$

$v_{\mathrm{sgs}}$ is related to a characteristic filter length (typically taken as proportional to the cube root of the local cell volume) and the local resolved scale strain rate magnitude. One indication of the lack of generality of this model is revealed by the wide range of values used for $C_{s}(0.09-0.18)$. In the present context, the main problem, however, is the model's behaviour near a wall, where it generates SGS viscosity wherever a velocity gradient exists, even in the laminar sub-layer. Whilst damping functions can be introduced, this is an ad-hoc modification and difficult to justify in general. Germano et al. [36] proposed a dynamic version which adjusts $\mathrm{C}_{\mathrm{S}}$ to local flow conditions, reducing the near wall value in laminar/transitional regions. The dynamic model has shown promising results - this was the closure adopted by both Sayadi and Moin [31] and Huai et al. [32] for example - but it is computationally expensive and requires numerical intervention (clipping) for stability. As an alternative, Nicoud and Ducros [37] proposed the Wall-Adapting Eddy Viscosity (WALE) model. This aims to recover proper near wall scaling $\left(O\left(\mathrm{y}^{3}\right)\right.$ for the eddy viscosity in near wall regions) without a dynamic procedure and allows $v_{\mathrm{sgs}}$ to approach zero in laminar regions. Investigations of Temmerman and Leschziner [38] for predicting separation from a curved surface showed that this model was best able to match the correct near wall scaling. The model is based on the square of the resolved velocity gradient tensor $\left(\bar{g}_{i j}=\partial \bar{u}_{i} / \partial x_{j}\right)$ :

$$
\begin{aligned}
& v_{s g s}=\left(C_{W} \Delta^{2}\right)\left[\frac{\left(L_{i j}^{d} L_{i j}^{d}\right)^{3 / 2}}{\left(\bar{S}_{i j} \bar{S}_{i j}\right)^{5 / 2}+\left(L_{i j}^{d} L_{i j}^{d}\right)^{5 / 4}}\right] \\
& L_{i j}^{d}=\frac{1}{2}\left(\bar{g}_{i j}^{2}+\bar{g}_{j i}^{2}\right)-\frac{1}{3} \bar{g}_{k k}^{2} \delta_{i j} \quad \bar{g}_{i j}^{2}=\bar{g}_{i k} \bar{g}_{k j}
\end{aligned}
$$

The use of $L_{i j}^{d}$ - the traceless symmetric part of the square of $\bar{g}_{i j}$ - means that the model is sensitive to both the strain and rotation rate of the turbulent structures which characterise the smallest resolved fluctuations. 
A relationship was proposed in [37] to provide the value of $C_{W}$ so that the same level of averaged SGS dissipation as the classical Smagorinsky model would be achieved. For a value of $C_{S}=0.1$ this implies $C_{W}=$ 0.35 and this was used in all simulations reported below.

It is important to stress that the rationale behind the current approach is that the code must be able to capture the unsteady laminar flow created by the DRE excited crossflow primary vortices without any SGS damping, but when the instability growth leads to breakdown into turbulence, the SGS model must provide sufficient dissipation to deal with energy passed down the cascade to the smallest resolved scale without damping in the near wall low Re regions; the method is perhaps best viewed as a hybrid Unsteady Laminar Flow/LES methodology.

\subsection{LES Code}

The computational code used here (DELTA) was developed at Loughborough University and has previously been applied to LES of impinging jets in crossflow (Li et al. [39]), to LES of subsonic jet aeroacoustics [40], and to LES of a full aircraft [41], also to the closely related problem to that considered here of LES of crossflow vortices on an infinite swept wing controlled by suction (Mistry et al. [42]). The code adopts a cell-centred, finite-volume, pressure-based method on a multi-block structured grid. Fluxes at the cell faces are constructed using second order central differencing for the convective velocity, pressure and viscous terms combined with a second order upwind (QUICK) scheme for convected terms. Temporal advancement is by a first order backward Euler implicit scheme or a low storage Runge-Kutta or fourth order (5-stage) scheme. Initial testing showed with a time step chosen sufficiently small to resolve the smallest scales that the two schemes produced identical results. However, the Euler implicit scheme was more robust to numerical problems induced by small and highly skewed cells, and so this was chosen for all calculations. The code uses a co-located flow variable arrangement, and to suppress odd-even decoupling, the standard Rhie and Chow [43] approach was added to all convective velocities. For LES it is essential that the smoothing term should be kept small to avoid unphysical dissipation of the resolved eddies and so the Rhie and Chow smoothing term was scaled by a factor of 0.1 for the calculations presented here. Parallelisation was achieved by mapping grid blocks to individual processors and updating interface regions using message passing. LES simulations were run in a non-dimensional scheme based upon freestream velocity $U_{0}=22.5 \mathrm{~m} / \mathrm{s}$ and a swept-wing chord

reference length $c=1.83 \mathrm{~m}$, giving $\operatorname{Re}_{c}=2.4 \times 10^{6}$. The time step was chosen such that the CFL number was less than 0.4 for the vast majority of the cells, resulting in a non-dimensional time step of less than $1.0 \times 10^{-05}$ and 5 inner iterations were run within each time-step. For a few small highly skewed cells this time step resulted in a $\mathrm{CFL}_{\max }$ of 0.95. The solver was run until 10 domain flow-through times had elapsed to allow the flow to develop; subsequently statistics were gathered by sampling sufficiently long to generate statistically stationary values (typically more than 20 flow-through times).

\subsection{Synthetic Eddy Model (SEM) for freestream turbulence}

In simulations of laminar/turbulent transition the specification of the freestream turbulence 
environment is clearly relevant. In some calculations below, freestream turbulence has been introduced using a Synthetic Eddy Model (SEM) to provide fluctuating freestream velocity boundary conditions. The SEM model was developed (Jarrin et al. [44], [45]) to provide LES inlet conditions. However, for the present application it was modified to provide physically realistic perturbations at a specified location in the freestream flow around the aerofoil (the 'SEM perturbation plane'). Essentially the SEM approach is applied to a selected zone within the flow domain rather than as an inlet condition. As previously discussed, the level of freestream turbulence in experimental studies was believed to be a major factor in explaining some of the discrepancies between the measurements. If freestream turbulence at a level measured in the experiment were introduced as a boundary condition at the simulation inlet plane (in the onset flow upstream of the swept wing), then to ensure the correct decay rate of this turbulence as the flow passed over the aerofoil, the turbulence integral length scale would need to be matched to experimental conditions as well as the turbulence intensity; the grid would also need to be made much finer in this region. Unfortunately, no experimental measurements of turbulence length scale are available for the flow cases considered here. Further, both stationary and traveling primary instabilities are thought to be influenced by freestream turbulence, and secondary instability growth and breakdown must also respond to the unsteady background disturbance. Without additional information, a full computational study of freestream turbulence is not possible. For the present investigation, it was decided to explore the response of secondary instability growth in the simulations to freestream turbulence. By generating the perturbations directly in the region of secondary instability observed without freestream turbulence this objective can be achieved. Clearly a full investigation of freestream turbulence is a subject for further study.

Figure 2 indicates the SEM perturbation plane selected in the present work for the simulations that included freestream turbulence (otherwise the freestream was assumed to be turbulence free). Note that Fig. 2 shows just a short spanwise strip of the aerofoil upper surface and contains two DRE cylinders close to the leading edge - the reasons for these choices is explained in Section 3. The SEM perturbation plane was chosen to be a mesh plane which was orthogonal to the freestream flow and located at $x / c=40 \%$ as shown in Fig.2; the perturbation plane was chosen at a position downstream of the roughness elements to ensure in this first exploration of freestream turbulence effects that the freestream disturbances would not influence the primary instability and initial growth stages. The plane was positioned approximately where secondary instabilities were observed to occur in the non-turbulent freestream simulation and in a region where nonlinear interactions were expected.

The first step in applying SEM is to create a (virtual) region (or box) which will contain the synthetic eddies and surrounds the SEM perturbation plane. Calculations within this box are used only to generate an unsteady freestream velocity field which is compatible with what is known about the turbulence conditions in the wind tunnel experiment. The finite set of points $S=\left\{\boldsymbol{x}_{1}, \boldsymbol{x}_{2}, \boldsymbol{x}_{3}, \ldots \ldots \ldots \boldsymbol{x}_{S}\right\}$ describes all the mesh points within the SEM perturbation plane. The dimensions of the box are determined by the SEM perturbation plane dimensions in the $y$ and $z$ directions; the $x$ direction size is determined by a parameter $\sigma$ which is related to the (expected) size of the largest turbulent eddies passing through the SEM perturbation plane. The 
minimum/maximum $x$ co-ordinates of this box were calculated via:

$$
x_{\min }=\min _{\boldsymbol{x}_{n} \in S}\left(x_{i}-\sigma\left(\boldsymbol{x}_{n}\right)\right) \quad \text { and } \quad x_{\max }=\min _{\boldsymbol{x}_{n} \in S}\left(x_{i}+\sigma\left(\boldsymbol{x}_{n}\right)\right)
$$

$\sigma\left(\boldsymbol{X}_{n}\right)$ is a user defined length scale, which was chosen to be the same value at all mesh points and set at $2 \%$ of the estimated boundary layer thickness at the SEM perturbation plane. Within the box the number of synthetic eddies considered is $N$; following Jarrin et al. [45] this is calculated from $N=V_{B} / \sigma$ where $V_{B}$ is the box volume. The SEM constructs the fluctuating velocity components at any point $\boldsymbol{x}_{n}$ in the SEM box from a sum of individual eddy contributions, thus:

$$
u_{i}^{\prime}\left(\boldsymbol{x}_{n}\right)=\frac{1}{\sqrt{N}} \sum_{k=1}^{N} a_{i j} \varepsilon_{j}^{k} f_{\sigma\left(x^{k}\right)}\left(\boldsymbol{x}_{n}-\boldsymbol{x}^{k}\right)
$$

where $\varepsilon_{j}^{k}$ is the intensity of the eddy located at $\boldsymbol{x}^{k}$ and $f_{\sigma\left(\boldsymbol{x}^{k}\right)}\left(\boldsymbol{x}-\boldsymbol{x}^{\boldsymbol{k}}\right)$ is the velocity distribution function of this eddy. $a_{i j}$ determines the magnitude of the velocity fluctuation, and is fixed by user-specified values of the Reynolds stress tensor $R_{i j}=\overline{u_{i}^{\prime} u_{j}^{\prime}}$ (Note - an overbar and prime here indicate time-average mean and fluctuation about the mean in a Reynolds decomposition sense) via the Cholesky decomposition:

$$
a_{i j}=\left(\begin{array}{ccc}
\sqrt{R_{11}} & 0 & 0 \\
R_{21} / a_{11} & \sqrt{R_{22}-a_{21}^{2}} & 0 \\
R_{31} / a_{11} & \left(R_{32}-a_{21} a_{31}\right) / a_{11} & \sqrt{R_{33}-a_{31}^{2}-a_{31}^{2}}
\end{array}\right)
$$

The velocity distribution function is selected again following Jarrin et al. [45]:

$$
\begin{gathered}
f_{\sigma\left(x^{k}\right)}\left(\boldsymbol{x}-\boldsymbol{x}^{k}\right)=\sqrt{\frac{V_{B}}{\sigma\left(\boldsymbol{x}^{k}\right)}} f\left(\xi_{x}\right) f(\xi) f\left(\xi_{z}\right), \quad \text { where: } \xi_{x}=\frac{\left(x-x^{k}\right)}{\sigma\left(\boldsymbol{x}^{k}\right)} \\
f(\xi)=\sqrt{\frac{3}{2}}(1-|\xi|) \quad \text { if }|\xi| \leq 1, \quad \text { and } \quad f(\xi)=0 \quad \text { otherwise }
\end{gathered}
$$

The locations of the synthetic eddies are initialized at a random distribution uniformly over the box volume, with intensities $\varepsilon_{j}^{k}$ equal to \pm 1 with equal probability to take one value or the other. The eddies are convected through the box such that their locations at each time step are calculated from $x^{k}(t+d t)=x^{k}(t)+U_{i} d t$ where $d t$ is the simulation time step and $\mathrm{U}_{\mathrm{i}}$ is the local mean velocity; if any eddy leaves the box, it is re-introduced at the box inlet plane at a random location within the plane.

The effect of this SEM treatment is that freestream turbulence at a specified level is introduced at the SEM perturbation plane; it is then convected downstream by the freestream velocity and is entrained into the boundary layer as this grows with downstream distance. Using this method, the three normal stress levels 
in the freestream were matched to the measurements provided by Hunt [14]:

$$
\sqrt{\overline{u^{2}}} / U_{0}=0.029 \% \quad \sqrt{\overline{v^{2}}} / U_{0}=0.04 \% \quad \sqrt{\overline{w^{2}}} / U_{0}=0.021 \%
$$

The hot-wire measurements in [14] were processed to remove the irrotational (acoustic) fluctuations that particularly influence the streamwise component, to isolate only the vortical (turbulence) contribution that is needed for the freestream boundary condition applied here. Full details of implementation and testing of the SEM approach are provided in Mistry [46]. Note that as soon as freestream turbulence is introduced, the sgs model becomes active for all cells in the freestream; to assess the impact of this the (averaged) sgs viscosity was examined. This showed that the peak value of $v_{s g s}$ was only $0.79 v_{m o l}$ and this occurred near the airfoil trailing edge and well within the boundary layer where the flow was fully turbulent. In the freestream cells downstream of $\mathrm{x} / \mathrm{c}=40 \%$ where freestream turbulence was introduced, the value of $v_{\text {sgs }}$ was between 0.0003-0.002 $v_{m o l}$. As a consequence of the small values of strain rate and $v_{\text {sgs }}$, the associated sgs stresses had a negligible effect on the introduced freestream turbulence.

\section{Test Case, Solution Domain and Mesh}

\subsection{Swept Wing Geometry and Flow Conditions}

Fig. 3 illustrates the wing and wind tunnel geometry (Hunt [14], Hunt and Saric [15]). The wing uses an ASU (67)-0315 aerofoil, specially designed to minimise attachment-line, Tollmien-Schlichting, and Goertler instabilities in order to allow crossflow disturbance to dominate transition. The wing has a $45^{\circ}$ sweep and is untapered. With an $x$-direction onset velocity $U_{0}$ of $22.5 \mathrm{~m} / \mathrm{s}$ and a swept wing chord $c=1.83 \mathrm{~m}$ this gives a chord-based Reynolds number $\operatorname{Re}_{c}=2.4 \times 10^{6}$. The wing was set at $-2.9^{0}$ incidence in order to achieve favourable pressure gradients over the upper surface of the wing. The wing span covers the whole width (zdimension) of the tunnel; the height (y-dimension) of the tunnel was $0.748 \mathrm{c}$, with the wing set just past the mid-tunnel location $(y=0)$ and profiled wall liners were used to ensure spanwise uniformity. The wing incidence placed the leading edge at $0.344 \mathrm{c}$ from the tunnel side wall and the trailing edge at $0.293 \mathrm{c}$. The experiments used a spanwise row of cylindrical-shaped roughness elements as DREs (diameter $3 \mathrm{~mm}$ ) located at $2.9 \% \mathrm{x} / \mathrm{c}$ from the leading edge on the suction surface, near the crossflow boundary layer neutral point. Both DRE amplitude $(\mathrm{k}=$ cylinder height in $\mu \mathrm{m})$ and spanwise spacing $(\lambda=$ spanwise wavelength in $\mathrm{mm})$ were varied. Hunt [14] used two values of $\lambda: 12 \mathrm{~mm}$ (critical), corresponding to the known most unstable mode of the laminar boundary layer (at the Reynolds number and angle of attack of the flow under consideration) and $6 \mathrm{~mm}$ (control), expected to delay transition.

The computational domain was initially chosen to correspond to the $x-y$ plane of the wind-tunnel, i.e. to include both upper and lower aerofoil surfaces ( $y$-dimension of $0.748 \mathrm{c}$ ); an axial length of $4 \mathrm{c}$ was used in the $\mathrm{x}$-direction. Choice of the spanwise z-dimension is important; a sufficiently large sector is needed to simulate the periodic array of DREs, but too large a dimension lowers resolution. DNS studies (Tempelmann et al. [47]) showed that two DREs were adequate to reproduce spanwise periodicity accurately; a z-domain size of $24 \mathrm{~mm}$ 
was thus used, with periodic boundary conditions set on the spanwise domain boundaries. This configuration is referred to as the 'full domain'. In order to make optimum use of the computational mesh, a second solution domain was exploited, referred to here as the 'suction surface domain'. The geometry of this domain was obtained by exporting from a statistically stationary solution on the full domain of the baseline flow (no DREs) the predicted stagnation streamlines upstream and downstream of the aerofoil (averaged over the spanwise direction). The DREs are located 18 cylinder diameters downstream of the leading edge and will only influence the upstream flow via their induced pressure field (penetrating perhaps $\sim 5$ diameters upstream), so their presence will have no impact on the upstream stagnation line. Figure 4 shows predicted mean streamwise $(U)$ velocity contours from a full domain simulation (containing 50 million cells, see below for details), with the stagnation streamlines identified. These lines upstream and downstream of the aerofoil were added to the suction surface geometry to form one boundary of the second computational domain

(Fig.5). Within the simulations these upstream and downstream surfaces were modelled as slip walls.

Solutions from both domains for the suction surface were compared and showed good agreement with each other and with experimental measurements (see Results section 4.1). All simulations presented here were therefore carried out with the smaller suction surface domain size to enable higher resolution to be achieved for a given amount of computational resource.

\subsection{Mesh Design}

The grid for the full domain (used for simulations of the baseline flow without DREs contained 50 million cells with 25 million on the suction surface; whilst that for the reduced size domain was increased to 95 million cells with all of these in the upper half of the full domain. Only the grid for the suction surface domain is detailed here since this was used for all quantitative analysis. A (half) C-grid was used around the aerofoil, extended upstream and downstream to reach flow inlet and outlet; Fig. 6(a) shows the C-grid block structure around the swept wing. To mesh the DRE cylinders an embedded O-topology was used. Figs. 6(b), 6(c) provide illustrations of the $x-y$ and $x-z$ meshes in the vicinity of a DRE cylinder, illustrating the fine mesh used to resolve the flow perturbation introduced by the cylinder. The fine mesh resolution of the boundary layer is also shown at the aerofoil leading edge in Fig. 6(d). All mesh generation was carried out using the ANSYS ICEMCFD 14.0 Hexa software.

The total mesh for the suction surface domain consisted of 190 blocks and 95 million hexahedral cells; the extra mesh was used to provide fine resolution of the suction surface, and in particular to provide better resolution in the immediate vicinity of the DRE cylinders to ensure accurate capture of the initial disturbance. 2250 nodes were placed along the aerofoil surface, 100 nodes were within the boundary layer in the wall normal direction, and 156 nodes were used spanwise. The spanwise node spacing implied a spatial resolution $\Delta z=0.15 \mathrm{~mm}$, compared with the experimental spanwise distance between profile measurement locations of $\Delta z=1.0 \mathrm{~mm}$. Fewer blocks could have been used to resolve the geometry but a larger number was chosen in order to be able to run on larger numbers of processors. Values of mesh size in wall units are $\Delta \mathrm{x}^{+}=8$ in the cylinder region and ranging from 30 at the leading edge through to 60 at the trailing edge. At the wall $\Delta \mathrm{y}^{+}=$ 
0.25 and across the span a uniform spacing of $\Delta z^{+}=11$ was used. These non-dimensional grid spacings correspond well to the maximum near wall cell size constraints recommended by Piomelli and Balaras [48] for well-resolved near wall simulations.

\section{Results}

\subsection{Baseline flow (no DREs)}

Figure 7 (a) shows predictions for the baseline flow aerofoil surface pressure distribution on both domains as well as experimental data (Hunt [14]). Solutions from full as well as suction surface domains agreed well with measured data. The measurements at two locations ('Upper' and 'Lower' ports) showed the flow was spanwise uniform. The predictions gave slightly higher values of suction than measured, but the gradient in both favourable and adverse regions, important for boundary layer stability and transition processes, was well matched. Velocity profiles in the laminar boundary layer in the vicinity of the DREs location $(x / c=10 \%)$ was compared with measured data in Fig. $7(b)$ and again shows close agreement.

\subsection{DRE receptivity - critical wavelength: $\lambda=12 \mathrm{~mm}$}

The spatial development of the crossflow vortices for the critical disturbance wavelength $\lambda=12 \mathrm{~mm}$ and a DRE height $\mathrm{k}=36 \mu \mathrm{m}$ is given in Fig. 8 , which shows contours of mean streamwise velocity at selected axial planes. The growth of the disturbance created by the DREs becomes visible at $x / c=20 \%$, where spanwise variations in boundary layer thickness appear. The spanwise wavelength becomes evident beyond $30 \%$, and at $40 \%$ distinct vortex structures are seen, with rollover evident at $50 \%$ and beyond.

The appearance of a rollover phenomenon is usually interpreted as the start of a non-linear growth region for the primary crossflow vortex, where the distorted mean flow profiles show slower growth (quasi-saturated amplitude) and display a low momentum region sandwiched between layers of high momentum fluid. This implies inflection points in the streamwise velocity profile (in both wall-normal and spanwise directions) quite high in the boundary layer (the mode shape begins to develop a double peak, see below). These regions are observed to lead to high frequency secondary instabilities. As described in the Introduction, these were studied by Malik et al. [22], who used a Non-linear Parabolised Stability Equation (NPSE) approach to identify two families of secondary instability depending on whether the energy input into the instability was fed by wall normal ( $y$-mode) or spanwise (z-mode) shear (a mixed $y$-z mode has also been observed). Detailed measurements of these have been presented by White and Saric [7]. It is these secondary instabilities which result in laminar/turbulent transition a short distance further downstream.

The shape and size of the crossflow vortices at $x / c=50 \%$ accords well with descriptions provided in the literature (Tempelmann et al. [47]). This flowfield clearly has boundary layer profiles which vary with spanwise location. This is the first measure of receptivity and is displayed in Fig. 9 at $x / c=15 \%$ for three DRE 
heights $\mathrm{k}=12 \mu \mathrm{m}$ (top), $24 \mu \mathrm{m}$ (middle) and $36 \mu \mathrm{m}$ (bottom) - note the axial velocity contours are here made non-dimensional using $U_{e}$ the local freestream velocity at the edge of the boundary layer. These contours show how the absolute level of disturbance at a given streamwise distance grows with DRE size. Experimental data are shown on the left and current predictions on the right; a clear $12 \mathrm{~mm}$ periodicity is seen, particularly in results from the largest DRE. Disturbance velocity profiles may be extracted from these results by subtracting the spanwise average profile from the local profile at each spanwise z-location. These profiles then quantify more readily the effects of roughness height, and are shown in Fig. 10. Good agreement between hot-wire measurements from [15] (left) and simulations (right) was obtained. Both experiments and simulations show some asymmetry between positive and negative deviation from the average profile; the deviations are greater in the experiments and are largest at the largest DRE height. For $\mathrm{k}=36 \mu \mathrm{m}$ for example, the maximum amplitudes of deviation from the average in the experiments for $\left(U-U_{a v g}\right) / U_{e}$ was $-0.068 \%$ and $+0.047 \%$, whereas in the simulation this was $-0.057 \%$ and $+0.054 \%$. One possible reason for this is the point mentioned above that the spanwise spacing in the measurements was $\sim 7$ times larger than in the simulations, and this may have influenced the evaluation of a true average in the measured data.

A stationary mode shape for the LES predicted disturbance was calculated using the same procedure as in the experiments by evaluating the RMS value of the perturbation about the spanwise mean at each $y$ distance from the wall; this estimate will therefore include all modes present, not just the initial input mode. Predictions for three values of $\mathrm{k}$ are shown in Fig. 11 at two streamwise locations, $\mathrm{x} / \mathrm{c}=15 \%$ and $20 \%$. In general excellent agreement with the hot-wire measurements of Hunt [14] can be seen. At $x / c=15 \%$ the stationary mode shapes show a single lobe with a maximum amplitude at $\sim 0.75 \mathrm{~mm}$ from the wall. In the experiments using the peak value as a parameter, a linear growth with DRE height was observed for $k=$ $12 \mathrm{~mm}$ and $24 \mathrm{~mm}$, but the growth rate does not quite scale linearly for $\mathrm{k}=36 \mathrm{~mm}$; it should be noted however that this measure includes all spanwise wavelengths present, not just the primary instability. In the LES predictions, for $k=24 \mathrm{~mm}$ and $36 \mathrm{~mm}$ agreement with measured data was close, but for the smallest DRE height the predicted amplitude was slightly greater than measured - grid resolution may be the cause (the same near cylinder mesh was used for all $k$ values), but small variations in DRE height due to manufacturing tolerance $(-\sim 2 \mu \mathrm{m}$ was quoted in [15]) will be more influential at small heights. At $x / c=20 \%$ the single lobe shape remains in both experiments and CFD, indicating no strong non-linear effects have yet begun; the amplitude of the largest DRE was underpredicted by $15 \%$, but the growth rate with axial distance was well captured. It should be noted that, at these stations, the peak fluctuations are at a distance from the wall that is already two orders of magnitude larger than the height of the DRE causing the disturbance.

As noted above, the stationary mode shape is a representation of the total disturbance signal, which may contain multiple modes. Following the practice adopted in the experiments, the spanwise disturbance signal at the height in the boundary layer corresponding to its peak value was spatially decomposed into modal amplitudes by taking a Fast Fourier Transform (FFT) of the signal. Figure 12 shows results for amplitude versus wavelength obtained for both $15 \%$ and $20 \%$ locations. At $15 \%$ the dominant mode excited is $\lambda=12 \mathrm{~mm}$, but a $6 \mathrm{~mm}$ wavelength is also observed; in fact at the smallest DRE height these two are approximately equal 
in amplitude. Using the peak amplitude as a measure, the growth rate of the primary instability does appear close to linear with DRE height. Further downstream at $x / c=20 \%$, the $12 \mathrm{~mm}$ wavelength has grown significantly. It has approximately doubled for all heights and this is still in the linear growth region for the primary instability; the smaller wavelength is still present but has not grown and has even decayed slightly for the larger $k$ values. These results indicate that the initial receptivity process for excitation in the critical wavelength mode has been well captured in the simulations, as well as its growth rate.

\subsection{DRE receptivity - control wavelength: $\lambda=6 \mathrm{~mm}$}

A similar analysis was carried out for the smaller (control) wavelength. An equivalent plot for $\lambda=6 \mathrm{~mm}$ for comparison with Fig. $9(\lambda=12 \mathrm{~mm})$ is shown in Fig.13, again for 3 DRE heights. The signature of 4 DREs can be clearly seen at this $x / c=15 \%$ location in the LES predictions; comparison with the experimental data for $k=$ $42 \mu \mathrm{m}$ shows that the maximum penetration of the peak regions created by the DREs matches quantitatively that seen in the measurements at $y=1.5 \mathrm{~mm}$, and the size of the low momentum region (where $U / U_{e}<0.2$ ) extending only $0.25 \mathrm{~mm}$ from the wall is also reproduced in the simulation.

The disturbance RMS profile and modal analysis for $\lambda=6 \mathrm{~mm}$ excitation is shown in Fig. 14 at the first measurement station. This indicates that a strong perturbation at the control wavelength has been generated. As in the $\lambda=12 \mathrm{~mm}$ case the smallest disturbance is underpredicted, again due to the difficulty in resolving this very small DRE on the grid. Note that in the experiments the profile is no longer a pure single lobe; the primary lobe has a peak at $0.9 \mathrm{~mm}$ from the wall, but there is evidence of a second lobe with a peak at $0.2 \mathrm{~mm}$. The simulations show no indication of this; the agreement with measurements for the primary lobe is good for the two larger DRE heights, but less good for the smallest, for the same reasons as mentioned previously. In all probability the near wall second lobe is a measurement error; extra lobes will appear due to non-linear growth (see below) but at this early axial station the growth is certainly in the linear regime and in any case second lobes due to non-linearity will appear in the outer region not near the wall. The amplitude plot for the simulation shows the clear presence of the $6 \mathrm{~mm}$ wavelength disturbance, although the growth rate is far from linear, with indications of a slowdown in growth. This suggests the disturbance is approaching saturation, perhaps nearing a region of decay; this is best analysed by examining the downstream development of the disturbance (see below).

Figure 15 displays the development of the overall disturbance for this case as before using contours of streamwise velocity on selected downstream planes (to be compared with the critical wavelength data in Fig. 8). Perhaps not so surprisingly given the discussion above, when the disturbance grows to a level where its signature can be seen in terms of discrete crossflow vortices, Fig. 15 shows that the $12 \mathrm{~mm}$ mode (2 vortices spanwise) now appears to dominate the flow behaviour, rather than the $6 \mathrm{~mm}$ mode (4 vortices spanwise). It is important to note that the behavior observed in the simulations is reflecting exactly what was observed in the experimental study. Hunt [14] and Hunt and Saric [15] suggested that the height of the DREs for this control wavelength case may have been chosen to be too large. This would cause the $6 \mathrm{~mm}$ mode to grow and decay quickly and the natural critical wavelength then takes over. It is clear that the $6 \mathrm{~mm}$ wavelength is 
excited and is present at $15 \%$ chord in both experiments and simulations, but disappears further downstream; to examine in more detail what is happening in this control wavelength case requires the development of the unstable stationary modes to be examined further downstream than was done in the experimental study, and this is described next.

\subsection{Evolution of instability modes - primary mode saturation and secondary instability}

In order to track the amplitude development of the instability modes it is usual in experiments to integrate rms disturbance plots such as those shown in Figs 11 and 14 over the near wall distance where these are non-zero to obtain an average strength for the instability at each downstream distance. A simpler approach has been taken here where the value of the peak amplitude has been used; Downs and White [19] have commented that whilst the integral evaluation leads to smoother curves, either practice leads to similar information on amplification ratios. Fig. 16 shows results extracted from the current LES predictions for peak modal amplitude development (Fig.16a) as well as rms disturbance mode shape (Fig. 16b) for the critical case $-\mathrm{k}=12 \mu \mathrm{m}$ and $\lambda=12 \mathrm{~mm}-$ and for downstream distances from $\mathrm{x} / \mathrm{c}=10 \%$ to $60 \%$. Although the DRE excitation is only for the $12 \mathrm{~mm}$ mode, as noted above in Fig. 12 the $6 \mathrm{~mm}$ mode also appears. The $6 \mathrm{~mm}$ mode grows slowly, remains weak and starts to decay at the last station. The critical unstable wavelength grows slowly at first (up to $x / c=20 \%$ ), then displays a region of rapid and linear growth up to $x / c=40 \%$ before growth rate slows and from $x / c=50 \%$ the amplitude decays. This general shape of development is exactly as predicted using NPSE analysis by Li et al [23] and also very similar to that shown in Fig. 11 of Downs and White [19] using the same swept wing model as Hunt and Saric [15] for the low freestream turbulence level of $0.02 \%$. The peak amplitude reached before the primary stationary mode begins to decay was measured at 0.11 in [19] which compares well with the value of 0.12 shown in Fig. 16a, especially given the different methods of amplitude evaluation. The end of the predicted linear growth region $(\sim x / c=40 \%)$ is confirmed by examining the rms mode shape plots in Fig. 16b. Single lobe plots are obtained up to $x / c=40 \%$ where the first sign of a convex curvature shape appears, and strong two-lobe shapes are obtained at both $50 \%$ and $60 \%$ chord, indicating clearly the occurrence of the roll-over phenomenon and strong mean flow profile distortion. The stationary mode disturbance decays between $50 \%$ and $60 \%$ as shown in both amplitude and mode shape results, indicating the appearance of the secondary instability region.

Repeating this analysis for the control wavelength $(\mathrm{k}=14 \mu \mathrm{m}, \lambda=6 \mathrm{~mm})$, the results obtained are provided in Fig.17. Once again Fig.17a shows that although only the $6 \mathrm{~mm}$ mode is excited by DREs, both $6 \mathrm{~mm}$ and $12 \mathrm{~mm}$ modes appear, with the former initially substantially stronger. As suspected by Hunt and Saric [15], even a DRE height of $14 \mu \mathrm{m}$ is too large because the control wavelength is only seen to grow up to $\mathrm{x} / \mathrm{c}=$ $20 \%$ after which it decays, to be overtaken by growth of the most unstable mode which behaves in a similar fashion as in Fig. 16 although with delayed growth, so it has only reached an amplitude of 0.09 at $x / c=50 \%$ rather than 0.12 when actively excited by DREs as in Fig.16. The mode shapes in Fig.17b support this showing the $12 \mathrm{~mm}$ mode still producing a roll-over lobe at the downstream stations. The LES predictions have thus confirmed the presumption made in Hunt and Saric [15] that in this flow the control mode was not effective. 


\subsection{Final transition to turbulence}

Results presented above have indicated that the receptivity process an subsequent instability growth are being captured well in the current simulations. Further analysis was therefore carried out to examine flow development downstream, where primary instability saturation occurs, followed by secondary instabilities and transition. All the simulations presented above have so far not considered any freestream turbulence effects; to include this in the examination of the final stages of transition to full turbulence, three cases have been analysed:

(i) $\mathrm{k}=12 \mu \mathrm{m}, \lambda=12 \mathrm{~mm}$ (critical) excitation with no freestream turbulence,

(ii) $\quad \mathrm{k}=12 \mu \mathrm{m}, \lambda=12 \mathrm{~mm}$ (critical) with freestream turbulence introduced using SEM,

(iii) $\quad \mathrm{k}=14 \mu \mathrm{m}, \lambda=6 \mathrm{~mm}$ (control) with SEM freestream turbulence.

Figures provided above have shown that for the first $40 \%$ of the wing chord, the near surface flow development is essentially dominated by the growth of the primary instabilities, with saturation (indicated by the appearance of roll-over in the individual crossflow vortices) starting at around $x / c=50 \%$. In this section therefore, attention is focussed on flow features in the aft region of the aerofoil where final transition to turbulence takes place. To visualise the development of the vortical structures in the flow, iso-surfaces of the $\lambda_{2}$ parameter are used as recommended by Jeong and Hussain [49].

Fig. 18 presents an instantaneous visualisation of the region between $\sim 70 \%$ chord and the aerofoil trailing edge for the flow of case (i). By experimenting with the value of the $\lambda_{2}$ iso-surface selected, it was established that for the first $50 \%$ of chord, a surface of $\lambda_{2}=-10$ captures the primary crossflow vortices best, showing these growing towards a saturated state and visible as 2 'tubes' propagating across the swept wing. Beyond $x / c^{\sim 70 \%}$ secondary instabilities and breakdown to turbulence may be identified. These events are illustrated in Fig. 18 for case (i) listed above (critical wavelength, no freestream turbulence), with the $\lambda_{2}$ isosurface coloured by spanwise (W) velocity. Two particular regions ( $A$ and $B$ ) have been identified upstream of $\sim 80 \%$ chord. Region A illustrates the start of destabilisation of the upper part of the crossflow vortices, with the appearance of secondary instabilities, triggered by the profile inflection points created by the roll-over. The second zone $B$ identifies a region of near zero axial velocity near the wall. The rapid deceleration of the near wall flow zone is accompanied by very high $\left(>15 \% \mathrm{U}_{0}\right)$ spanwise velocity and this has been used to highlight zone $B$ in Fig. 18. Note that zone B starts just aft of the pressure minimum at $x / c=71 \%$ (see Fig. 7) and significantly upstream of the zone A onset of crossflow vortex secondary instability breakdown. Zone B is co-incident with the high adverse pressure gradient region, which creates conditions for rapid deceleration and possible separation. Fig. 19 indicates that a small, thin separation region is in fact predicted to occur by the current LES solution. Fig.19 displays a zoom-in of near wall instantaneous axial velocity contours at 5 spanwise locations across the solution domain. The region between $x / c=75 \%$ and $85 \%$ is shown and indicates a clear zone of negative axial velocity may be observed. This varies in axial extent both in time and across the span; the $\mathrm{max} / \mathrm{min}$ extent of this is indicated in Fig.19 as $78 \%-82 \%$. This causes a breakdown into 
turbulence via the Tollmien-Schlichting instability, with large scale eddy structures appearing near the wall starting around $x / c=83 \%$ as shown in Fig.19. The development of this turbulence in the near wall region and its spread across the whole boundary layer occurs rapidly as indicated very clearly in Fig. 18 with multiple highly unsteady large scale eddies appearing and a large increase in boundary layer thickness occurring. Thus, whilst both crossflow vortex secondary instability breakdown and the near wall separating flow ae both possible triggers to final transition to turbulence, in the simulation of case (i) it seems clear it is the latter which is the primary cause.

Figure 20 shows a similar image as in Fig.18 for the case (ii) flow (critical wavelength DRE but now with freestream turbulence), for the same region of the aerofoil and with the same parameters. The same two possible sources of transition are evident as in case (i). The crossflow vortex secondary instability region seems more marked and breakdown of this starts slightly earlier. In the region marked A there is visible evidence of the breakup of the primary vortex tubes. The near-wall instability region is less evident, but examination of the near-wall axial velocity behaviour as done in Fig. 19 for case (i) shows that separation is still predicted to occur in case (ii). In the present simulations the freestream turbulence has thus predominantly affected the flow by delaying the rapid deceleration zone and its consequent near-wall instability. This allows the crossflow vortex secondary instability to develop further. It is thus predicted that the route to transition even in case (ii) is from the near-wall source and this will be confirmed below when discussing Fig. 23. This result is contrary to that observed in experiments, where increasing freestream turbulence is observed to de-stabilise the boundary layer and move transition forwards. One possible explanation is that the length scale at which the freestream turbulence is introduced by the current SEM treatment is different to that occurring in the experiment. Unfortunately no measurements of the turbulence integral scale in the freestream were made, so it is difficult to analyse this odd result of the present simulations any further. Clearly more measurements and simulations of freestream turbulence effects on transition are required.

Further insight into transition in the case (ii) flow as resolved in the current simulations is gained from Fig. 21, which shows contours of time-averaged axial velocity (right) and time-averaged axial RMS fluctuations (left) for the case (ii) flow at axial locations from $50 \%$ to $80 \% \mathrm{x} / \mathrm{c}$. The region shown is for the full width of the solution domain $(24 \mathrm{~mm})$ and for the $12 \mathrm{~mm}$ just above the suction surface. At $50 \% \mathrm{x} / \mathrm{c}$ the discrete crossflow vortices can be seen to be just at the start of roll-over. The peak fluctuation amplitude is less than $5 \%$ of $U_{0}$ and is concentrated near the low momentum upwelling region, where the flow is being squeezed by the roll-over. At $60 \% \mathrm{x} / \mathrm{c}$ roll-over is well underway and the high fluctuation region and its peak amplitude have both grown; the higher turbulence is also causing noticeable smearing of the velocity contours. Note that the highest turbulence region is present in the near wall flow rather than in the shear layer on the edge of the crossflow vortex; this is consistent with the view stated above that transition in the case (ii) simulation is still driven by near wall rather than crossflow vortex breakdown. This process continues until by $80 \% \mathrm{x} / \mathrm{c}$ the peak amplitude of turbulent fluctuations is now greater than $10 \%$ and the near wall flow is almost two-dimensional, with only the outer edges of the vortex now visible. This seems to confirm that even with freestream turbulence the transition has its origin in near-wall processes. 
If the case (iii) flow ( $6 \mathrm{~mm}$ control wavelength, with freestream turbulence) is considered, Fig. 22 shows a different picture than for cases (i) and (ii). The evidence in Fig. 22 is that the near wall flow breakdown is much less evident, whereas the outer regions of the crossflow vortex breakdown seem to be of increased intensity and to continue much further downstream. It seems possible from Fig. 22 that in the case (ii) flow the final transition to turbulence may be driven primarily by the crossflow vortex.

The interpretations given above on transition are reinforced if the contours of skin friction coefficient $C_{f}$ on the wing surface are examined; these are shown in Fig. 23 for all 3 flow cases. The case (i) flow (Fig. 23 top) shows alternating high/low values of $C_{f}$ occurring between/beneath the crossflow vortices entering on the left. From $60 \% \mathrm{x} / \mathrm{c}$ onwards skin friction begins to decrease as the vortices spread and decelerate and there are signs of waves appearing in the primary vortices, which is interpreted as evidence of the beginning of secondary vortex breakdown in the outer region. Beyond $\sim 70 \% \mathrm{x} / \mathrm{c}$ rapid deceleration of the near wall flow occurs across the entire span and a region of near zero $C_{f}$ is seen, this is followed at $x / c=78 \%$ by transition to turbulent flow across the whole span. The two-dimensional nature of the transition line in this region where an adverse pressure gradient is expected suggests that this is a Tollmien-Schlichting induced transition.

The middle image in Fig.23 allows interpretation of the effect of freestream turbulence (case (ii)). Since turbulence will act to increase the entrainment of high momentum freestream fluid, the $C_{f}$ signature of the primary vortices shows evidence of higher near wall velocities between the vortex tubes. This enables the primary vortices to persist longer downstream against the adverse pressure gradient, the region of near stagnation flow is no longer evident and the transition location has moved downstream to $x / c=80 \%$, with transition still initiated in the decelerating near wall flow, although the transition line is less two-dimensional than in case (i), showing that the crossflow vortex breakdown is more established in case (ii).

The control wavelength case (iii) $C_{f}$ plot is shown at the bottom of Fig. 23. The signature of the two crossflow vortices is again evident in the $C_{f}$ plot for this case, although the delayed development of the $12 \mathrm{~mm}$ wavelength vortices as indicated in Fig. 17 means that in the upstream part of Fig.23 the stationary vortex amplitude is smaller than in cases (i) and (ii) and the wall shear stress is therefore lower. Nevertheless, this also means that the maximum amplitude of the primary vortices is further downstream. This enables the vortices to persist longer and this leads to a wedge-shaped burst of turbulence as expected for classical crossflow transition. Thus, only in the simulation of case (iii) does the crossflow vortex trigger transition, in the simulations of cases (i) and (ii) this results more from the near-wall separating flow. Note that this causes the transition to move further upstream (to $\sim 72 \%$ chord) in case (iii) than in case (ii). Although this forward shift of transition with the control DREs is not the desired outcome to reduce drag, it is noteworthy that the same upstream shift was observed in the experimental measurements of Hunt [14]; in the present simulations this is due to the different reasons for transition driven by either near wall processes or crossflow vortex breakdown. 


\section{Summary and Conclusions}

The work described above has examined the ability of an LES code-based simulation with a simple eddy viscosity SGS model to capture all phases of the crossflow-induced transition process on a swept-wing. The prime objective of the study was to examine the trends observed in simulations for receptivity, downstream growth of DRE-excited primary instabilities (covering linear, non-linear and saturation regions), secondary instability, and final transition to fully turbulent flow, and where possible contrast these with trends observed in experiments. Comparison with experimental measurements validated the approach for the receptivity phase. Two DRE spanwise wavelengths were considered: critical and control; it was found in the simulations that the control case displayed an even earlier transition than the critical case, reflecting what was also observed in experiments. The effect of freestream turbulence was explored by using an SEM LES inlet condition approach to introduce turbulence into the freestream fluid entrained into the boundary layer. For the swept-wing case of Hunt and Saric [15] studied here, where the laminar region on the aerofoil suction surface (even in the baseline case without DREs) extended beyond the pressure minimum, examination of the breakdown into full turbulence revealed two possible causes. The first was due to transition in a region of stagnating flow near the wall, and the other due to a classical secondary instability and formation of a turbulent wedge in a primary vortex. In terms of future work, several areas are possible. The effect of small differences in height of the DREs due to manufacturing variability was something noted in the measurements. It would be of interest to use the simulation approach developed here to understand this better. If the mesh in the immediate DRE vicinity was carefully refined, simulations should be able to determine the consequences of variations in roughness height and even shape. Finally, further study of freestream turbulence effects would also be very useful; the present simulation implied that increasing freestream turbulence delayed transition, whereas the opposite has been observed in measurements. One possible explanation for this discrepancy is that the length scale at which turbulence was introduced in the freestream was not matched between simulations and experiments. Currently, although wind-tunnel data exist on the impact on transition of increasing the amplitude of turbulence in the freestream, but no data exist on the integral length scales at which these various amplitudes occurred. More detailed measurements to guide parallel simulations would be beneficial.

\section{Acknowledgements}

The authors would like to thank the Flight Physics Department at Airbus (Filton) for provision of supercomputer time and financial support of the project. In addition some simulations have been carried out using HPC Midlands, which is funded by UK EPSRC Grant EP/K000063/1. 


\section{References}

1. Flightpath 2050: Europe's vision for aviation, High Level Group on Aviation Research, (2011).

2. Arnal, D., Archambaud, J. P.: Laminar-turbulent transition control: NLF, LFC, HLFC, Advances in LaminarTurbulent Transition Modelling, VKI Lecture Series, Brussels, Belgium, (2009).

3. Green, J., E.: Laminar flow control - back to the future? AIAA paper 2008-3738 (2008).

4. Poll, D. I. A.: Some observations of the transition process on the windward face of a long yawed cylinder. J. Fluid Mech. 150, 329-356 (1985).

5. Saric, W. S., Reed, H. L., White, E. B.: Stability and transition of three-dimensional boundary layers. Ann. Rev. of Fluid Mech. 35, 413-440 (2003).

6. Radeztsky, R. H. Jr, Reibert, M. S., Saric, W. S.: Effect of isolated micron-sized roughness on transition in swept-wing flows, AIAA J., 37, 370-1377 (1999).

7. White, E. B., Saric, W. S.: Secondary instability of crossflow vortices. J. of Fluid Mech. 525, 275-308 (2005).

8. Dagenhart, J., R., Saric, W., S.: Crossflow stability and transition experiments in swept-wing flow. NASA TP 1999-209344 (1999).

9. Saric, W. S., Carrillo, R. B., Reibert, M. S.: Leading edge roughness as a transition control mechanism, AIAA Paper. 98-0781 (1998).

10. Carpenter, A. L., Saric, W. S., Reed, H. L.: Roughness receptivity in swept wing boundary layers experiments. Int. Jnl. Eng. Sys. Modelling and Simulation. 2, 123-128 (2010).

11. Reibert, M. S., Saric, W. S., Carrillo, R. B., Chapman, K. L.: Experiments in non-linear saturation of stationary crossflow vortices in a swept-wing boundary layer. AIAA Paper. 96-0184 (1996).

12. Borodoulin, V., I., Ivanov, A., V., Kachanov, Y., S., Roschektaev, A., O.: Receptivity coefficients at citation of crossflow waves by freestream vortices in the presence of surface roughness. J. Fluid Mech. 716, 487 -527 (2013).

13. Kurian, T., Fransson, J., H., Alfredsson, P., H.: Boundary layer receptivity to freestream turbulence and surface roughness over a swept flat plate. Phys. Fluids. 23, 034107 (2011).

14. Hunt, L. E.: Boundary layer receptivity to 3D roughness arrays on a swept wing, PhD Thesis, Texas A\&M Univ., USA (2011).

15. Hunt, L. E., Saric, W.S.: Boundary layer receptivity of three-dimensional roughness arrays on a swept wing, AIAA Paper. 2011-3881 (2011).

16. Carrillo, R. B.: Distributed roughness effects on stability and transition in swept-wing boundary layers. M. Sc. Thesis, Arizona State University, USA (1996).

17. Saric, W. S., Carpenter, A. L., Reed, H. L.: Passive control of transition on three-dimensional boundary layers with emphasis on discrete roughness elements. Phil. Trans. Ry. Soc. Lond. A 369, 1352-1364 (2011) 
18. Lovig, E., N., Downs, R., S., White, E. B.: Passive laminar flow control at low turbulence levels. AIAA J. 52, 1072-1075 (2014).

19. Downs, R., S., White, E., B.: Freestream turbulence and the development of crossflow disturbances, J. of Fluid Mech. 735, 347-380 (2013).

20. Riedel, H., Sitzmann, M.: In-flight investigations of atmospheric turbulence. Aerospace Science and Technology 2, 301-319 (19998).

21. Reshotko, E., Saric, W. S., Nagib, H. M.: Flow quality issues for large wind tunnels, AIAA-97-0325.

22. Malik, M. R., Li, F., Choudhari, M. M., Chang, C.-L.: Secondary instability of crossflow vortices and sweptwing boundary-layer transition. J. of Fluid Mech. 399, 85-115 (1999).

23. Li, F., Choudhari, M., Chang, C-F., Streett, C., Carpenter, M.: Computational modelling of roughnessbased laminar control on a subsonic swept-wing. AIAA J., 49, 520-529 (2011).

24. Haynes, T., S., Reed, H., L.: Simulation of swept-wing vortices using non-linear parabolised stability equations. J. Fluid Mech. 305, 325-349 (2000).

25. Menter, F., R., Langtry, R., B., Likki, S., R., Suzen, Y., B., Huang, P.H., Voelker, S.: A correlation-based transition model using local variables: Part I - model formulation. ASME J. of Turbomachinery 128, 413422 (2006).

26. Wasserman, P., Kloker, M.: Transition mechanisms induced by travelling crossflow vortices in a threedimensional boundary layer. J. Fluid Mech. 485, 67-89 (2003).

27. Wasserman, P., Kloker, M.: Transition mechanism in a 3D boundary layer with pressure gradient changeover. J. Fluid Mech. 530, 265-280 (2005).

28. Hosseini, S. M., Tempelmann, D., Hanifi, A., Henningson, D. S.: Stabilisation of a swept-wing boundary layer by distributed roughness elements, J. Fluid Mech. Rapids, 718, R1 (2013).

29. Kurz, H., B., E., Kloker, M., K.: Receptivity of a swept-wing boundary layer to micron-sized discrete roughness elements. J. of Fluid Mech. 755, 63-82 (2014).

30. Schlatter, P.: Large Eddy Simulation of transition and turbulence in wall bounded shear flow. PhD thesis, Swiss Federal Institute of Technology, Zurich (2005).

31. Sayadi, T., Moin, P.: Large Eddy Simulation of controlled transition to turbulence. Physics of Fluids 24, 114103 (2012).

32. Huai, X., Joslin, R., Piomelli, U.: Large Eddy Simulation of boundary layer transition on a swept wedge. J. of Fluid Mech. 381, 357-380 (1999).

33. Bonfigli G, Kloker M., Wagner, S.: Three-dimensional boundary layer transition induced by superposed steady and travelling crossflow vortices. In - High Performance Computing in Science and Engineering 2002. Springer, Berlin/ Heidelberg (2003)

34. Sagaut, P.: Large Eddy Simulation for Incompressible Flows: An Introduction. Springer Berlin/Heidelberg, Germany (1998).

35. Smagorinsky, J.: General circulation experiments with the primitive equations - I. The basic experiment. Monthly Weather Review, 91, 99-164 (1963). 
36. Germano, M. N., Moin, P., Cabot, W. H.: A dynamic sub grid scale eddy viscosity model. Physics of Fluids A: Fluid Dynamics 3, 1760 -1765 (1991).

37. Nicoud, F. and Ducros, F.: Sub-grid-scale stress modelling based on the square of the velocity gradient tensor, Flow Turb. \& Comb. 62, 183-200 (1999).

38. Temmerman, L., Leschziner, M., A.: Large Eddy Simulation of separated flow in a streamwise periodic channel constriction. Proc. of $2^{\text {nd }}$ Int. Symposium on Turbulence and Shear Flow Phenomena 393-404 (2002).

39. Li, Q., Page, G., J., McGuirk, J., J.: Large Eddy Simulation of twin impinging jets in cross-flow. The Aeronautical Journal, 111, 195-206, 2007.

40. Afsar, M., Z., Hynes, T., P., Dowling, A., P., McMullan, W., A., Pokora, C, Page, G., J., McGuirk, J.,J.: Jet Noise: Acoustic Analogy informed by Large Eddy Simulation, AIAA J., 48, 312-1325 (2010).

41. Page, G. J., McGuirk, J. J.: Large Eddy Simulation of a complete Harrier in ground effect. The Aeronautical Journal 113, 99-106 (2009).

42. Mistry, V. I., Page, G. J. McGuirk, J. J.: Large Eddy Simulation of crossflow vortices on an infinite swept wing, AIAA Paper 2012-2694 (2012).

43. Rhie, C. M., Chow, W. L.: Numerical study of the turbulent flow past an aerofoil with trailing edge separation. AIAA J. 21, 1525-1535 (1983).

44. Jarrin, N., Benhamadouche, S., Laurence, D., Prosser, R.: A synthetic eddy method for generating inflow conditions for large-eddy simulations. Int. J. of Heat \& Fluid Flow 27, 585 - 593 (2006).

45. Jarrin, N., Prosser, R., Uribe, J.-C., Benhamadouche, S., Laurence, D.: Reconstruction of turbulent fluctuations for hybrid RANS/LES simulations using a synthetic eddy method. Int. J. of Heat \& Fluid Flow 30, $435-442$ (2009).

46. Mistry, V. I.: Simulation and control of crossflow vortices, PhD Thesis, Loughborough Univ. UK, (2014).

47. Tempelmann, D., Schrader, L. U., Hanifi, A., Brandt, L., and Henningson, D. S.: Swept wing boundarylayer receptivity to localised surface roughness. J. of Fluid Mech. 711, 516 - 544 (2011).

48. Piomelli, U., Balaras, P.: Wall layer models for large eddy simulations. Annual Review of Fluid Mechanics 34 349-374 (2002).

49. Jeong, E., Hussain, F. On the identification of a vortex. J. of Fluid Mech., 85, 69 - 94 (1995). 


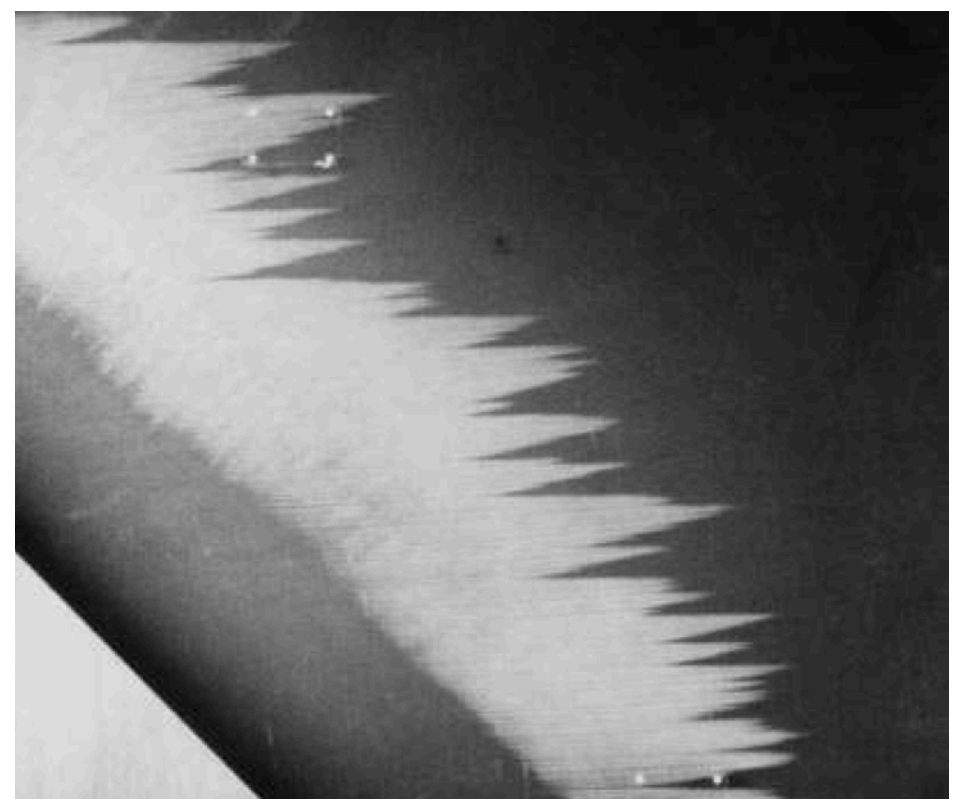

Figure 1: Typical laminar/turbulent transition line for crossflow instability-induced transition (Dagenhart and Saric [8])

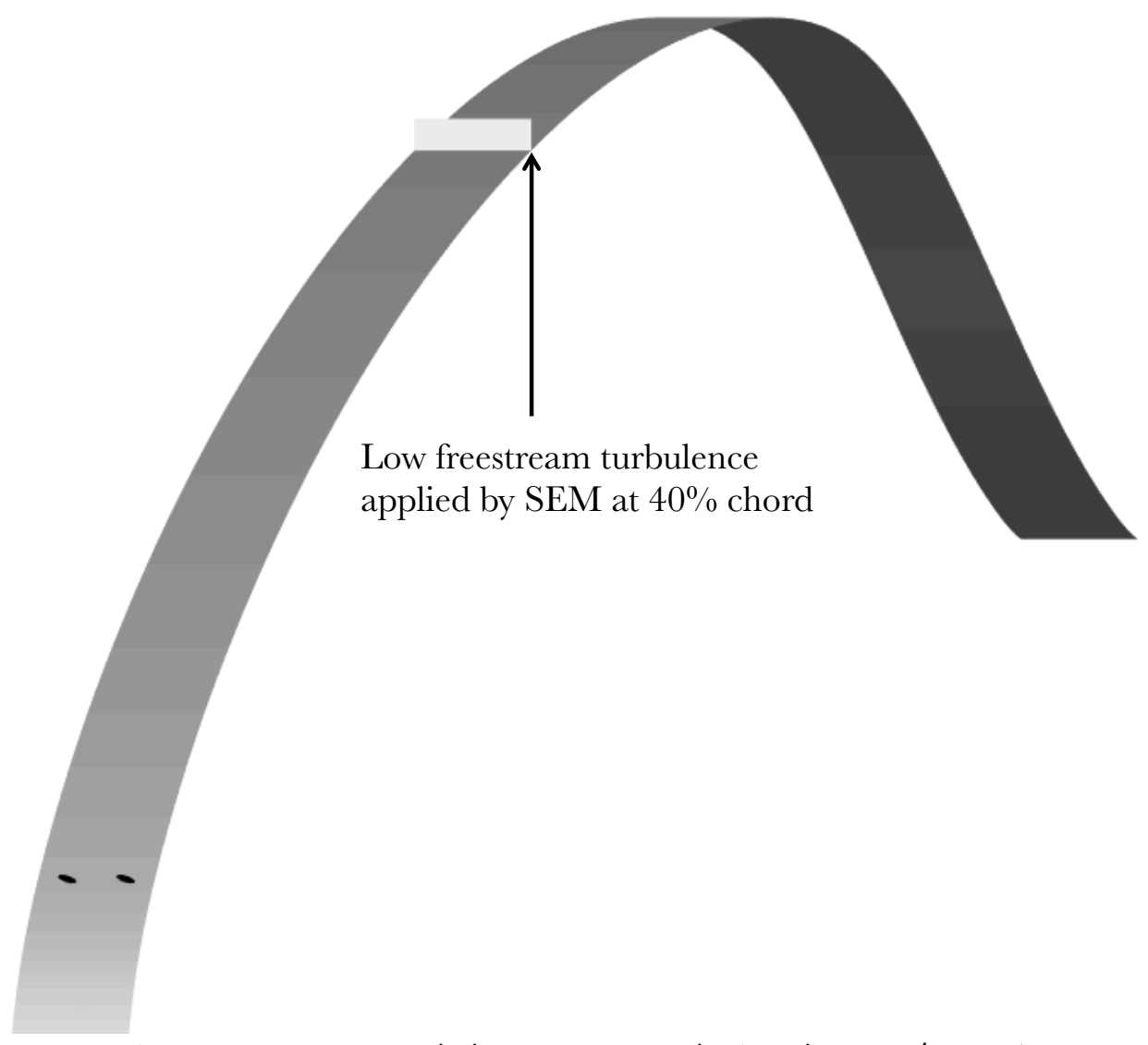

Figure 2: Freestream turbulence SEM perturbation plane at $x / c=40 \%$ 

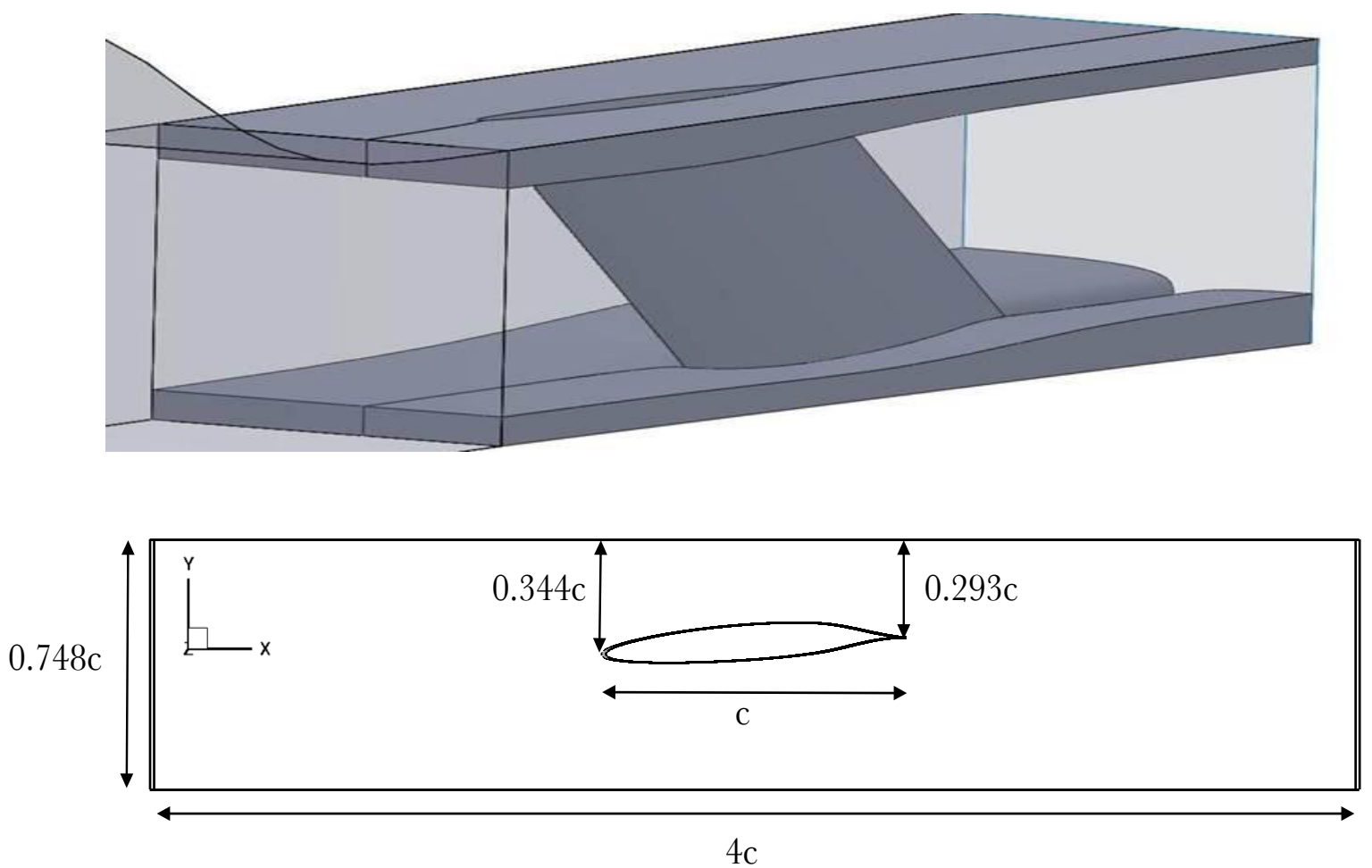

Figure 3: Top - Wind tunnel geometry [14], bottom - longitudinal slice on z = 0 plane

\section{Stagnation Streamline}

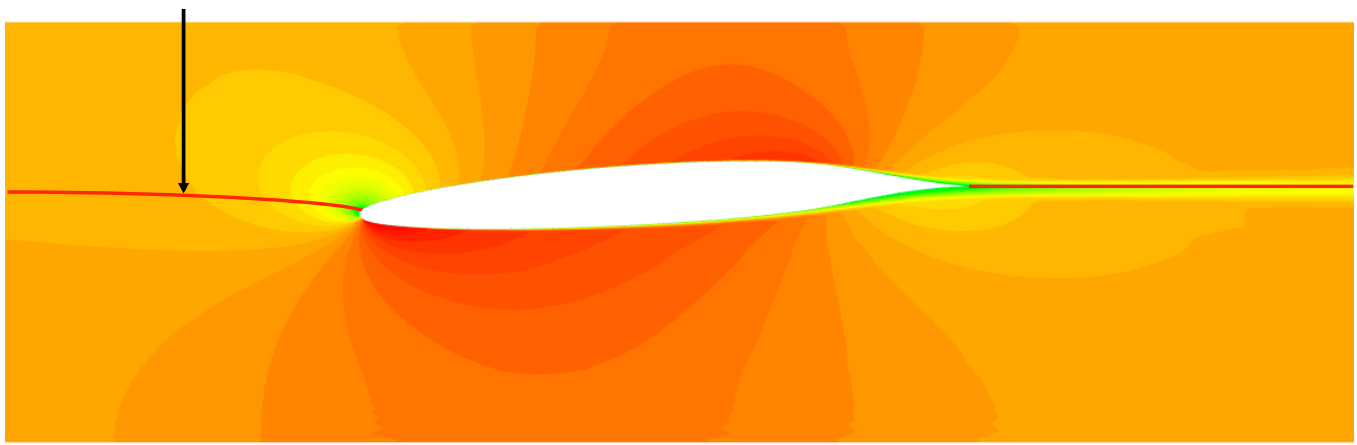

$\begin{array}{llllllllllllll} & \text { V Velocity: } \quad 0 & 0.1 & 0.2 & 0.3 & 0.4 & 0.5 & 0.6 & 0.7 & 0.8 & 0.9 & 1 & 1.1 & 1.2\end{array}$

Figure 4: Full domain LES solution for baseline flow

$4 \mathrm{c}$

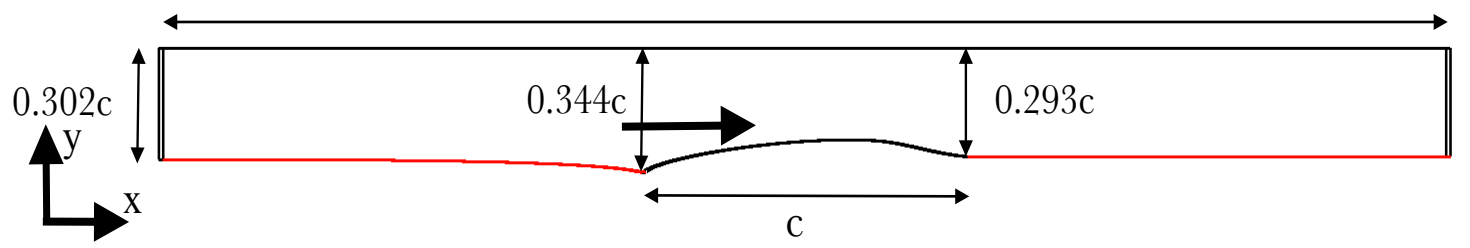

Figure 5: Suction surface LES solution domain 


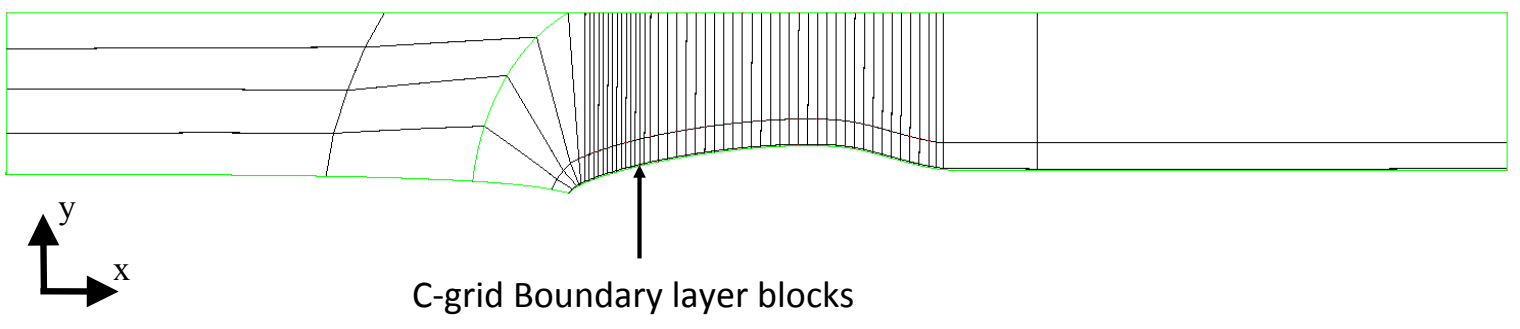

(a)

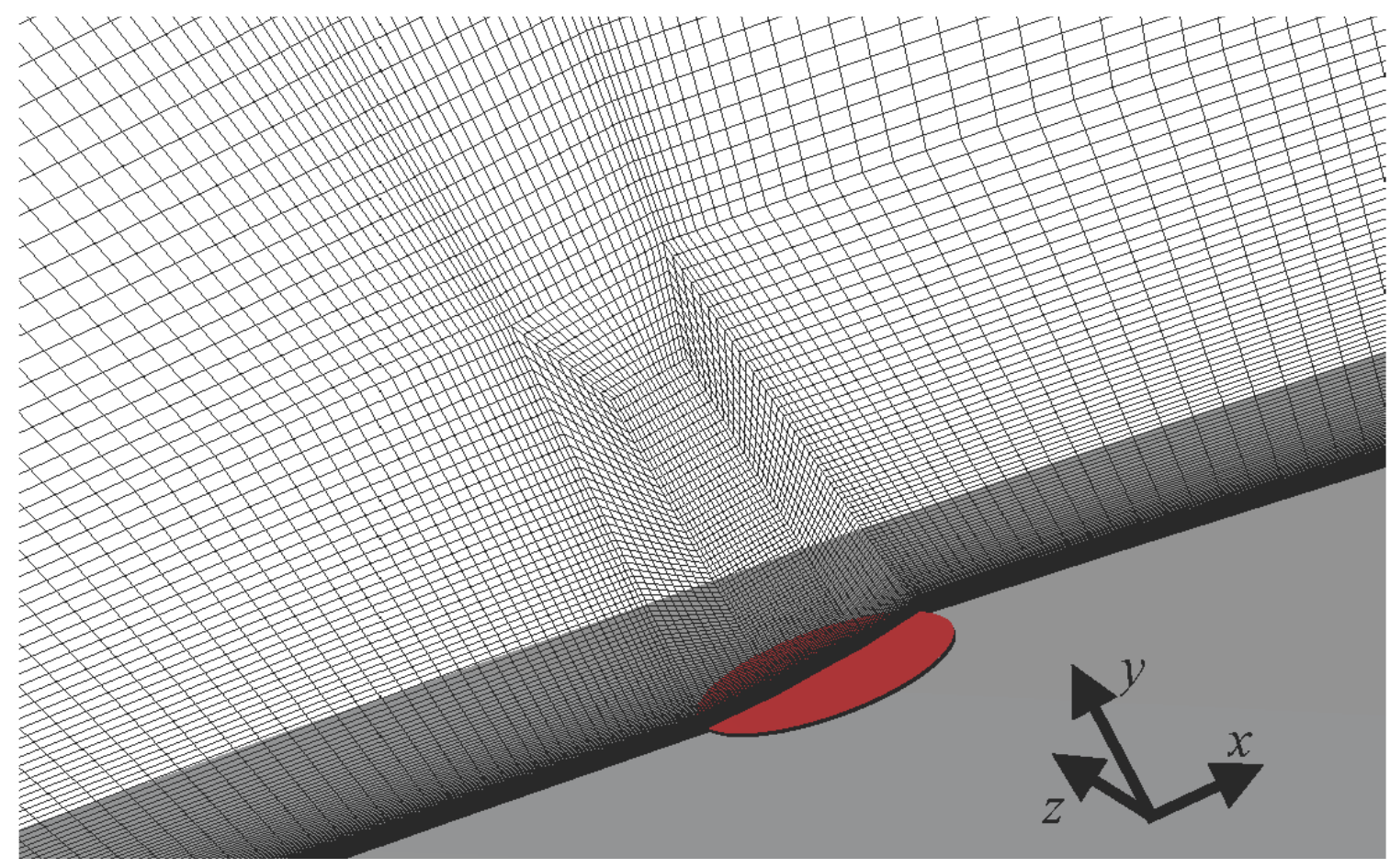

(b) 


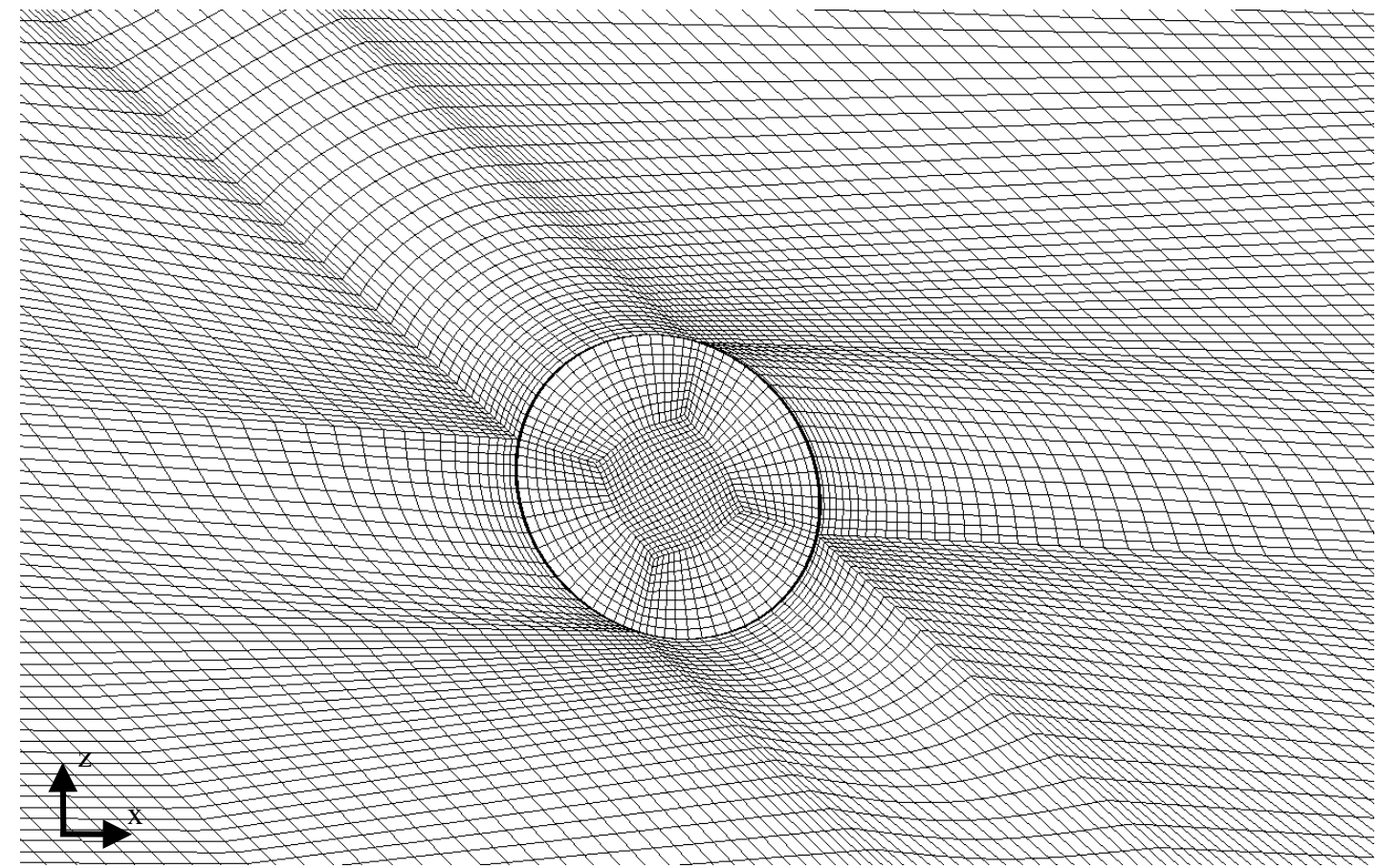

(c)

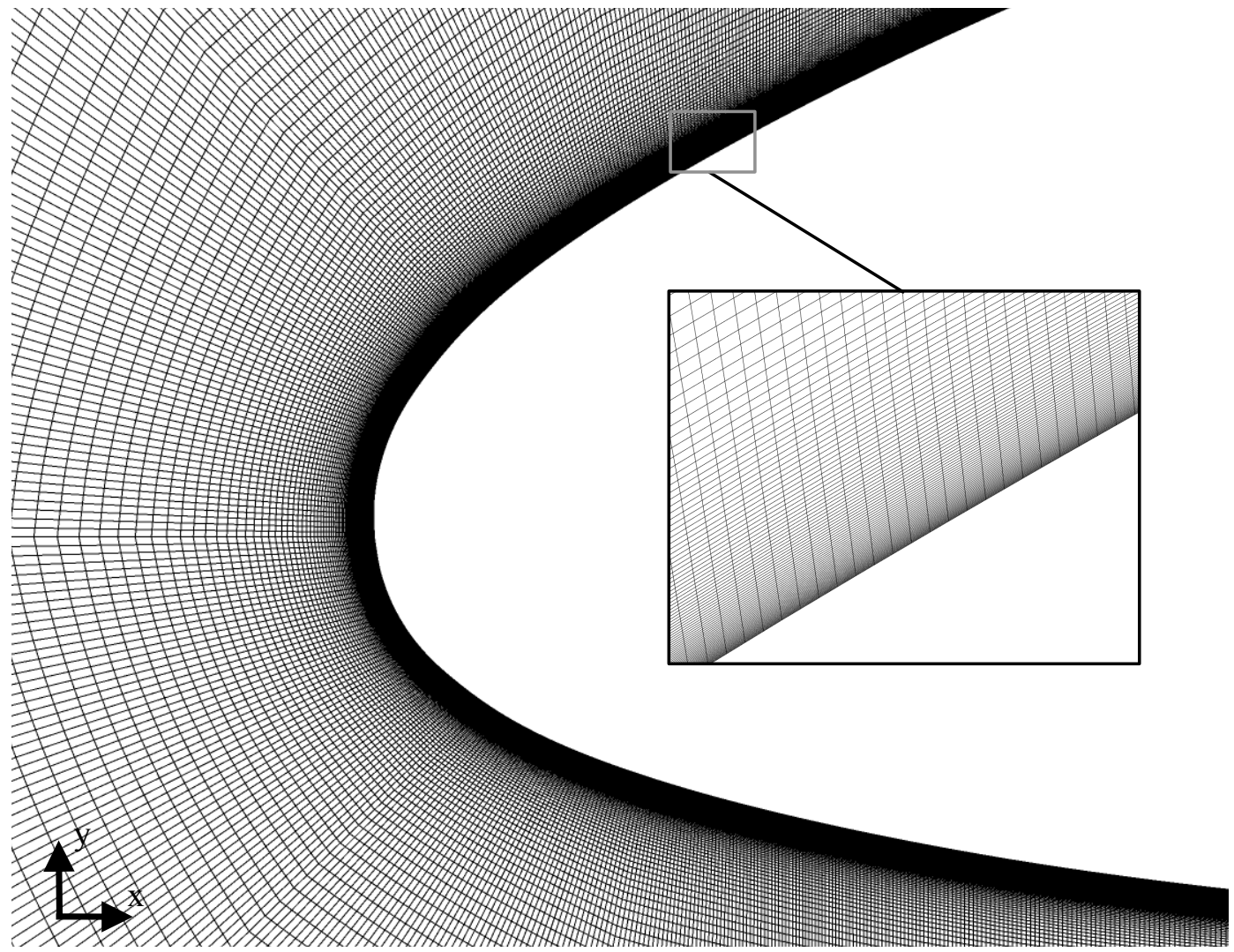

(d)

Figure 6: (a) Mesh blocking around aerofoil, mesh planes in vicinity of DRE cylinders (b) and (c) and at aerofoil leading edge (d) 


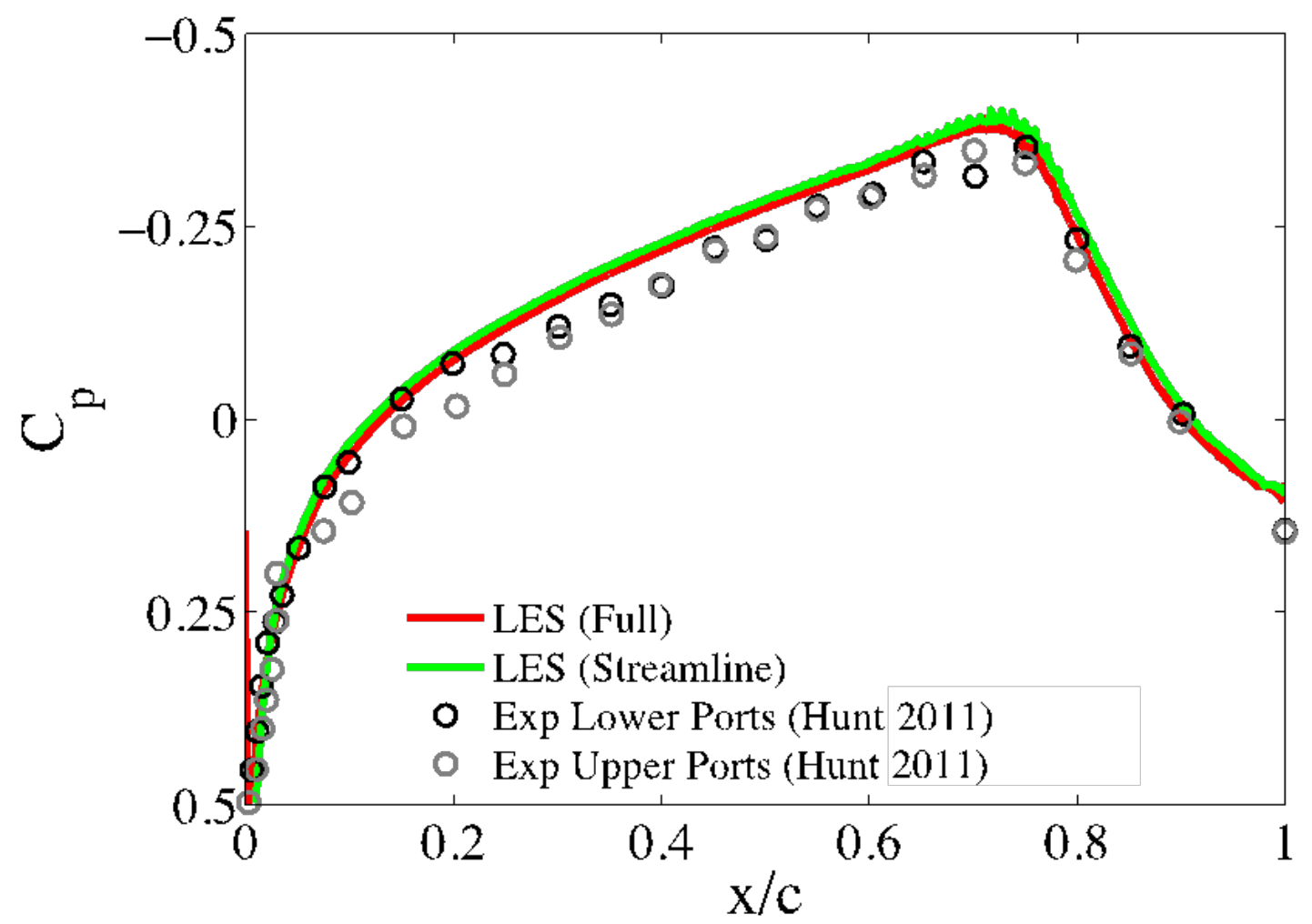

(a)

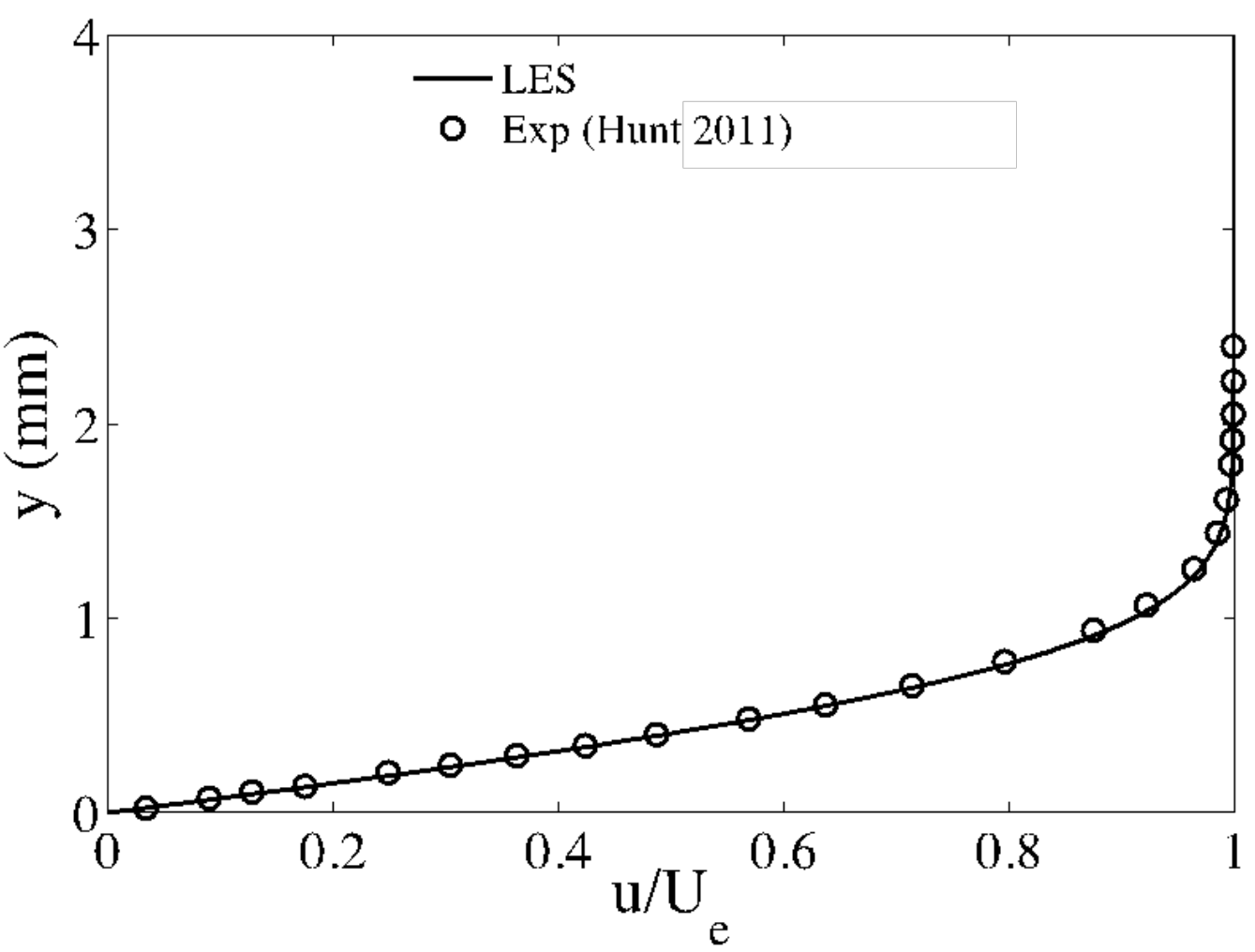

(b)

Figure 7: (a) Pressure coefficient and (b) velocity profile at $x / c=10 \%$ - comparison with expt. data [15] 


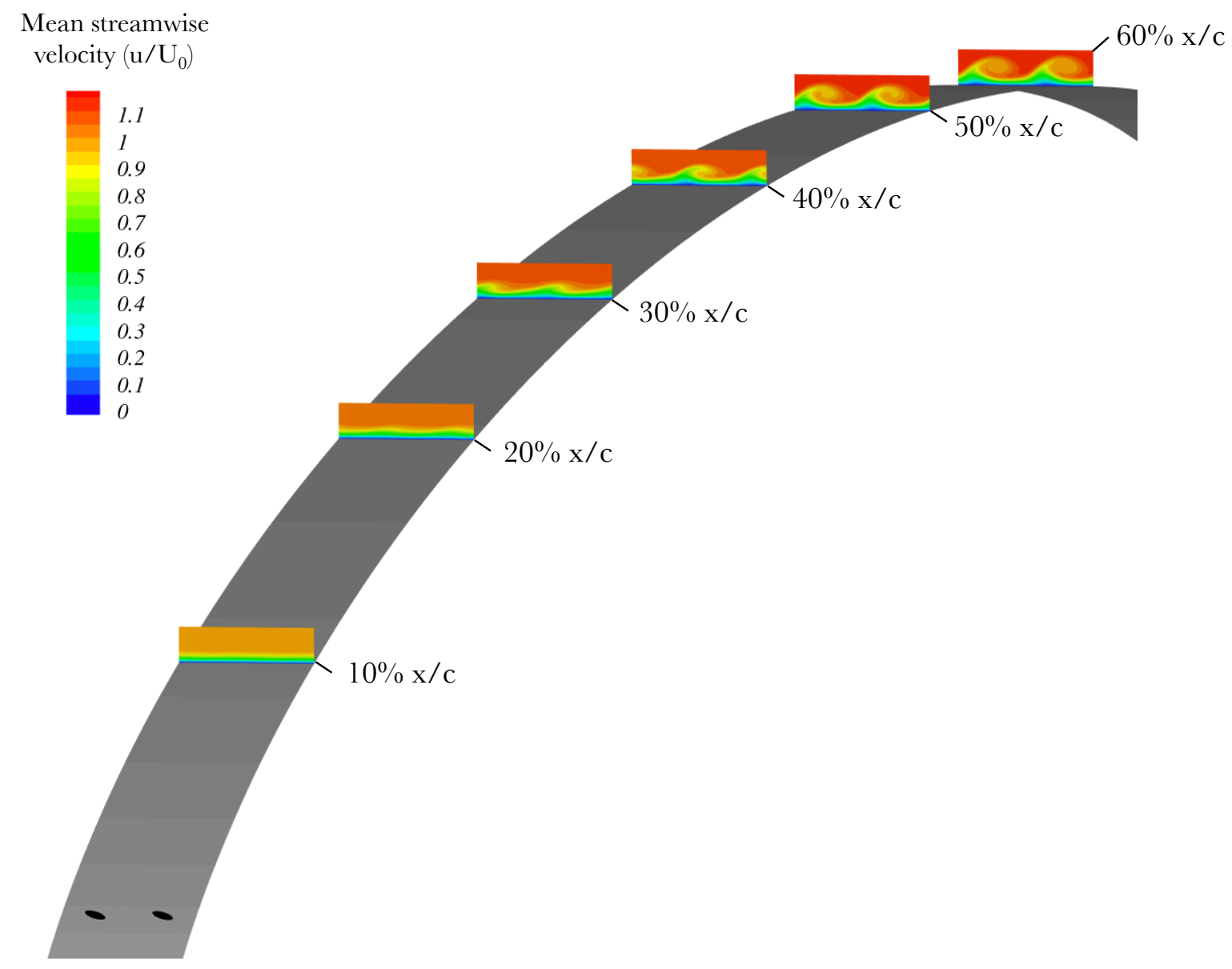

Figure 8: Axial velocity contours, $\lambda=12 \mathrm{~mm}, k=36 \mu \mathrm{m}$ 
Experiment
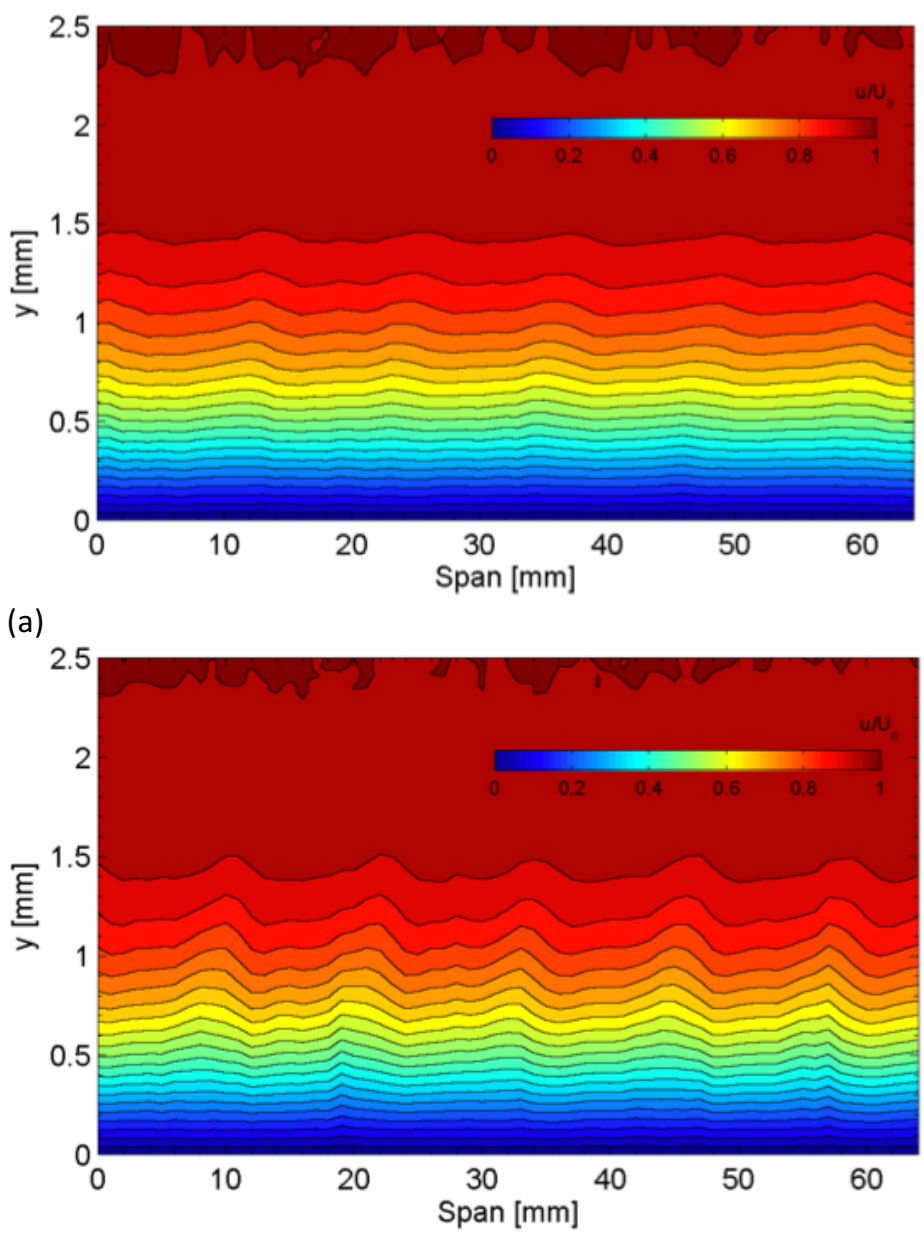

(b)

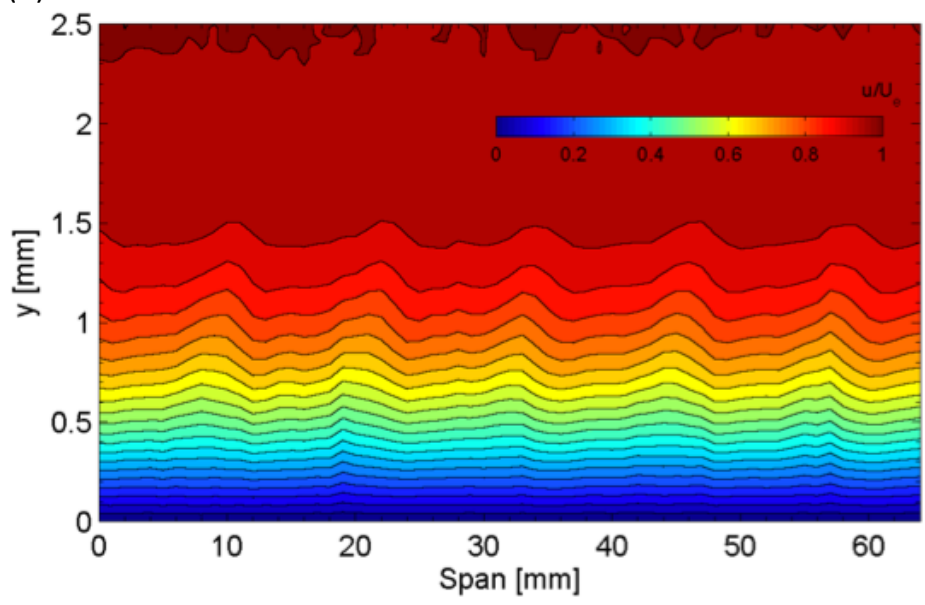

$\underline{\text { LES }}$
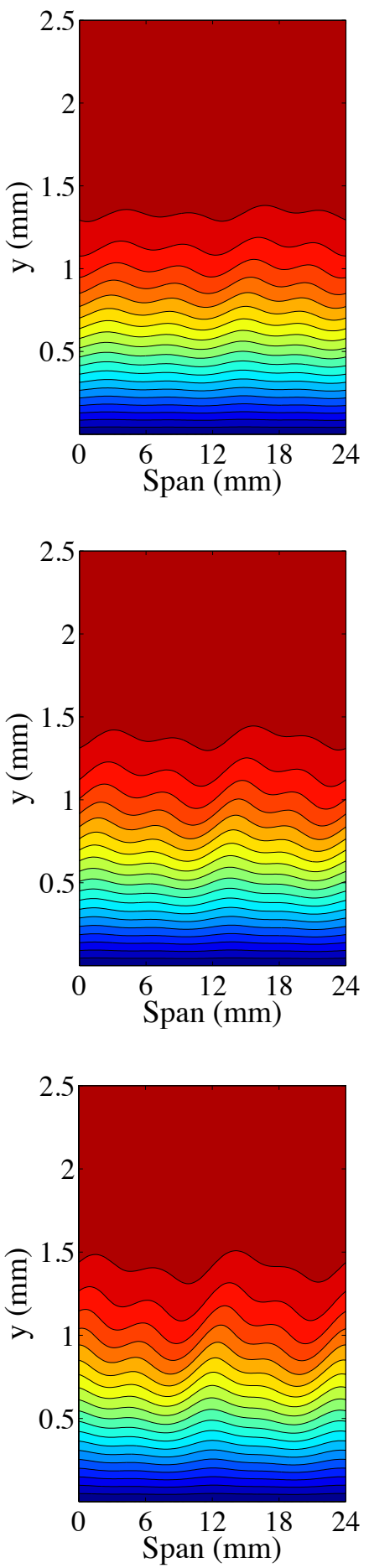

(c)

Figure 9: Contours of $u / U_{e}$ at $x / c=15 \%$ for 3 roughness heights, $\lambda=12 \mathrm{~mm}$, a) $k=12 \mu \mathrm{m}$, b) $k=24 \mu \mathrm{m}, \mathrm{c}) \mathrm{k}=36 \mu \mathrm{m}$; experiment (left), simulation (right) 

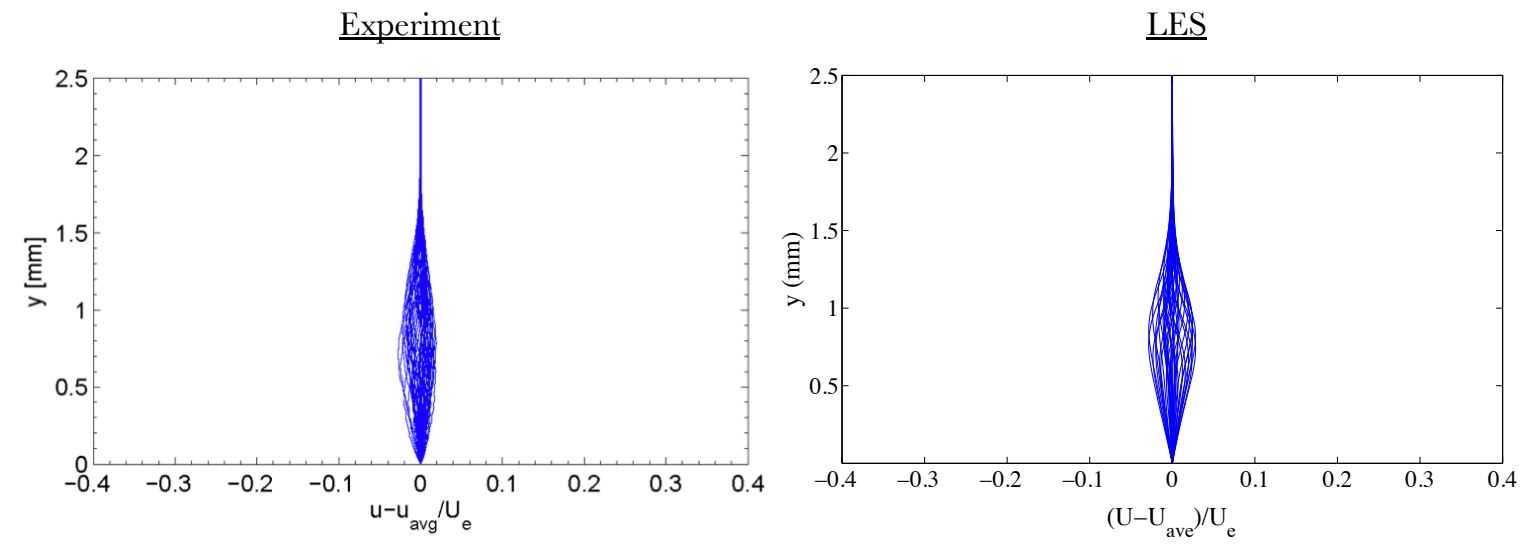

(a)
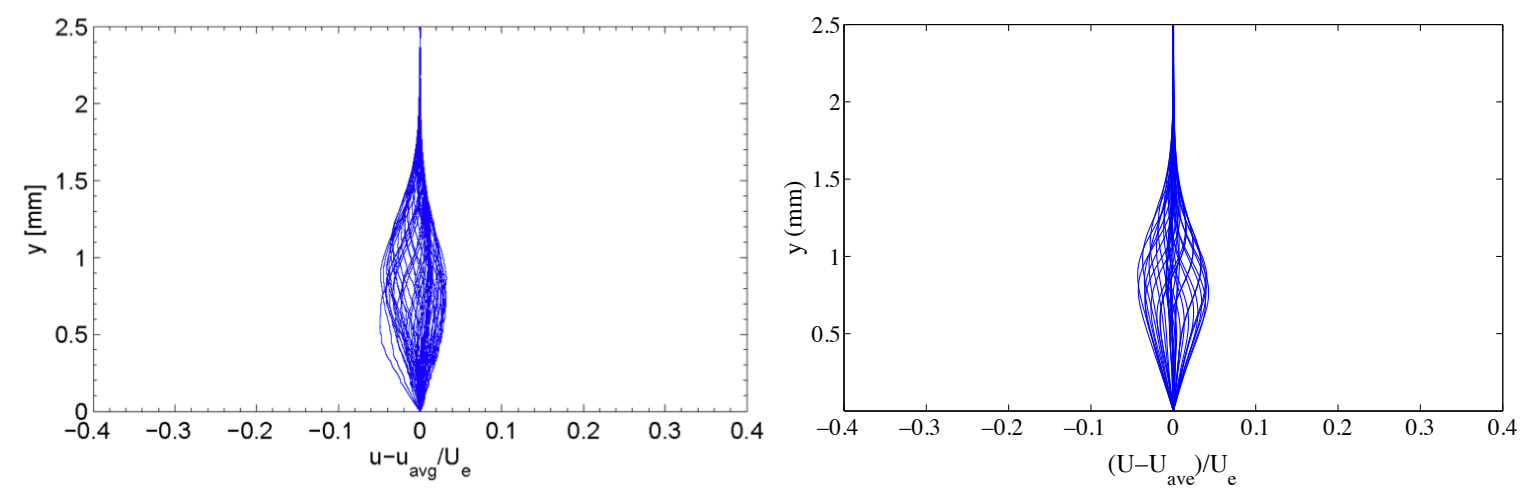

(b)
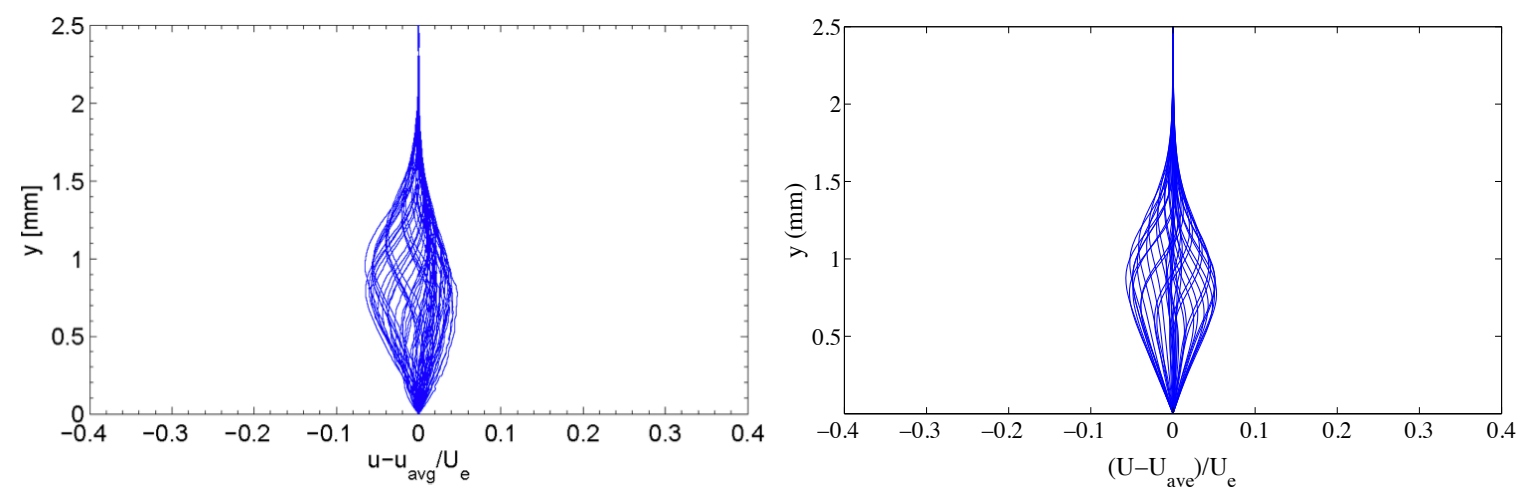

(c)

Figure 10: Disturbance profiles: $x / c=15 \%$, comparison of expts. [15] (left) \& simulation (right) for $\lambda=12 \mathrm{~mm}$ a) $k=12 \mu \mathrm{m}$, b) $k=24 \mu \mathrm{m}, \mathrm{c}) \mathrm{k}=36 \mu \mathrm{m}$ 


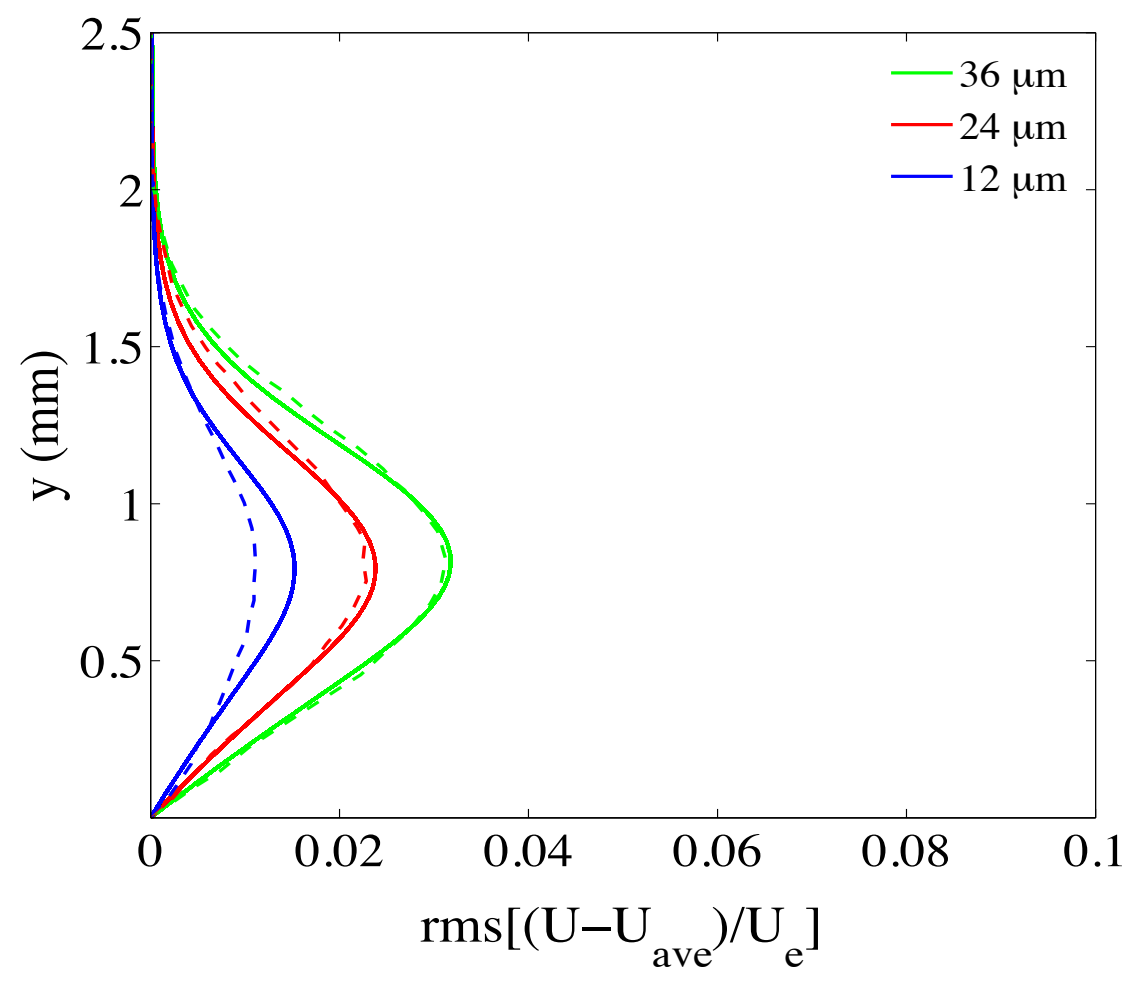

(a) 


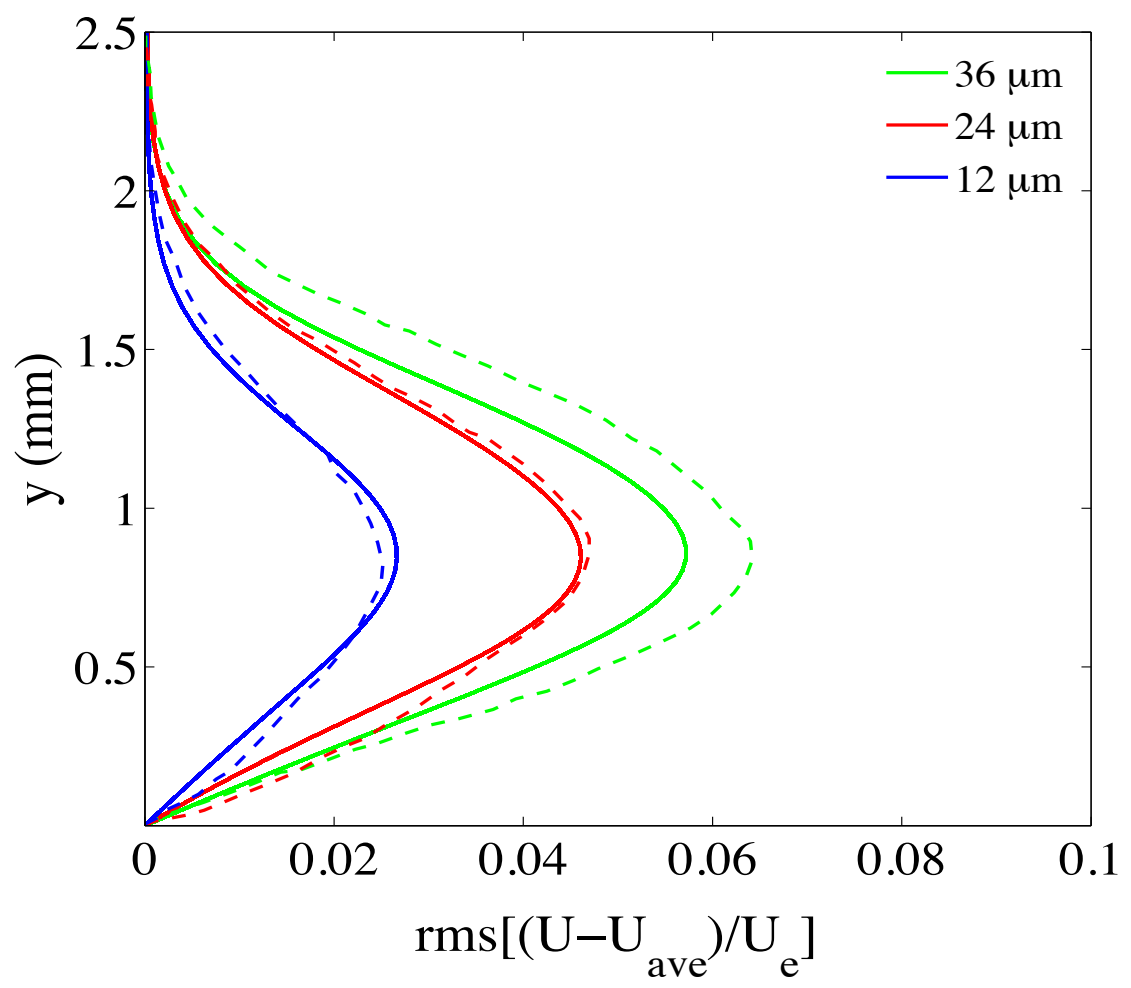

(b)

Figure 11: Disturbance mode shape, (a) x/c=15\%, (b) 20\%; solid lines-CFD; dashed lines - Expts. [15]

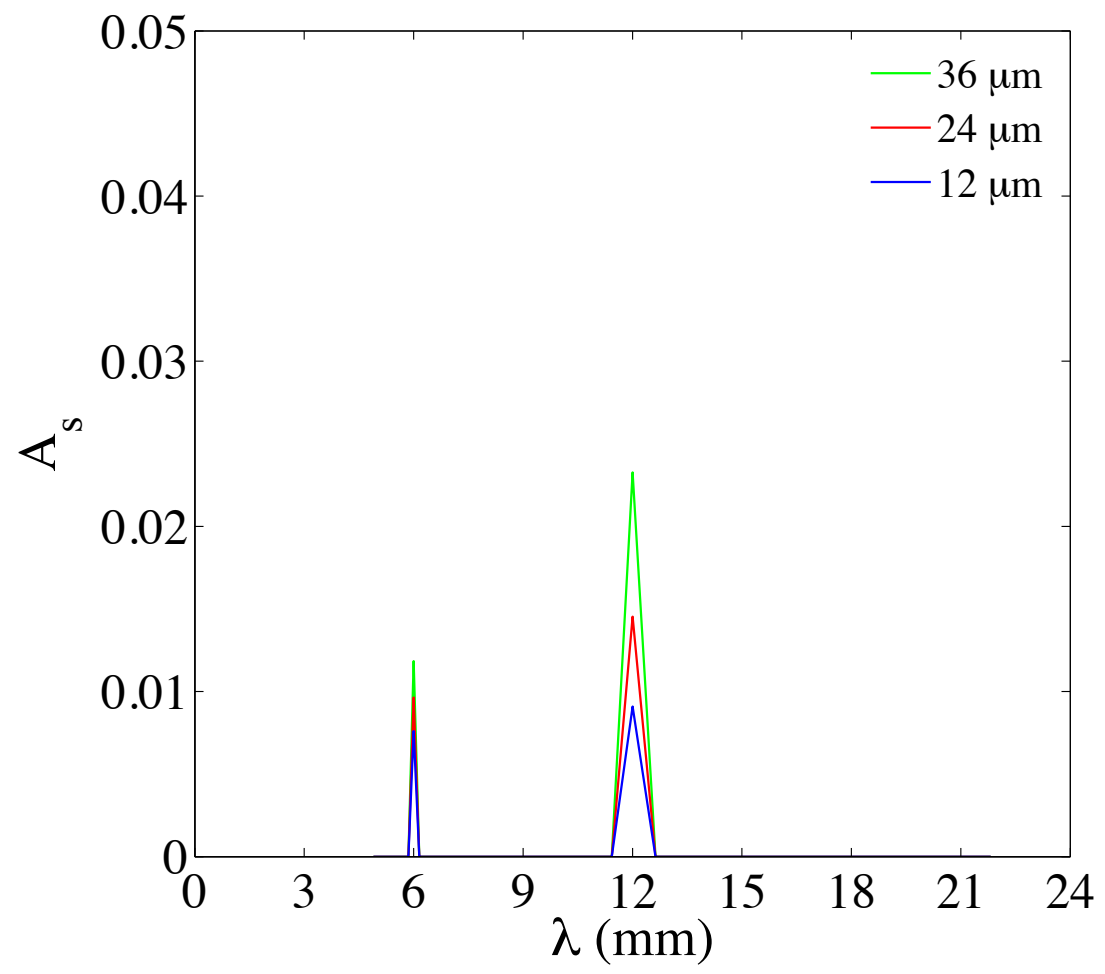

(a) 


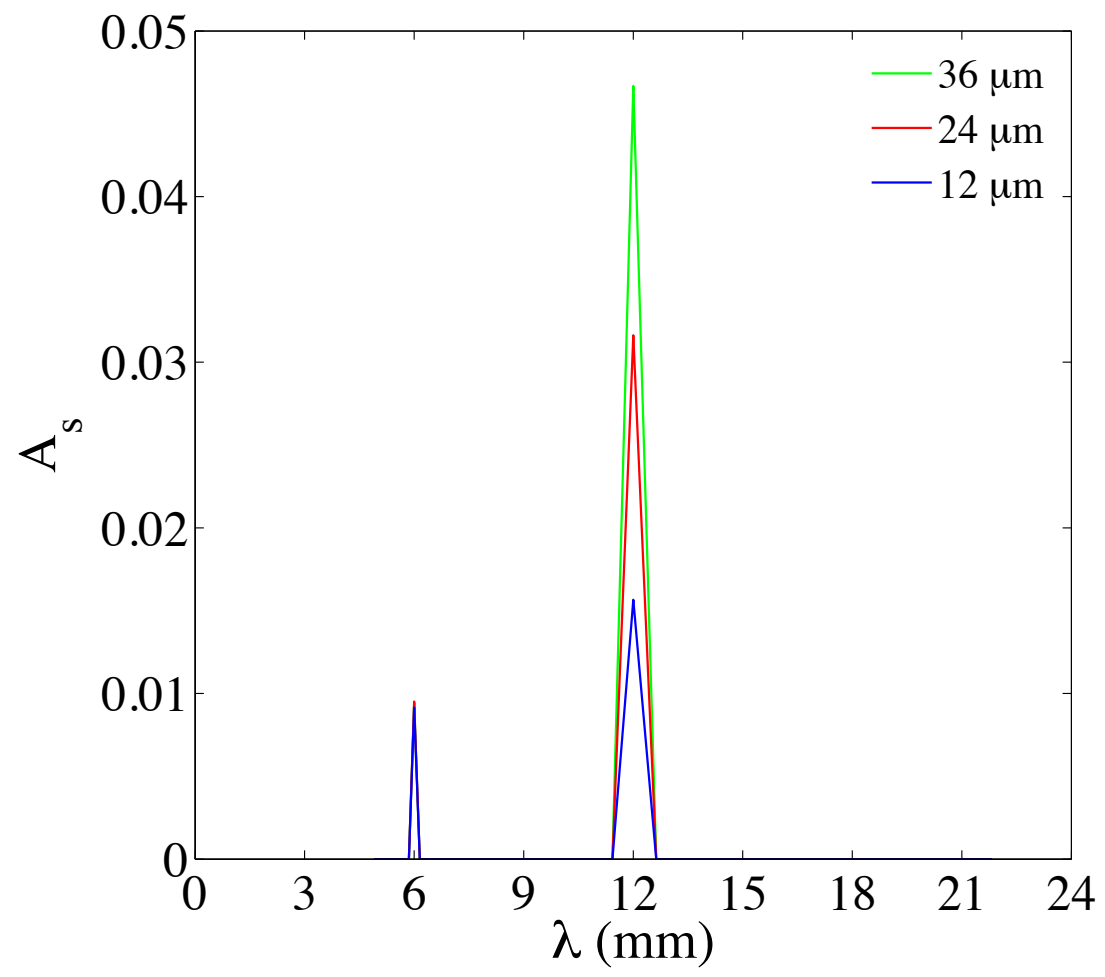

(b)

Figure 12 Amplitude spectra of disturbance mode at (a) $x / c=15 \%$, (b) $20 \%$ for CFD predictions

Experiment

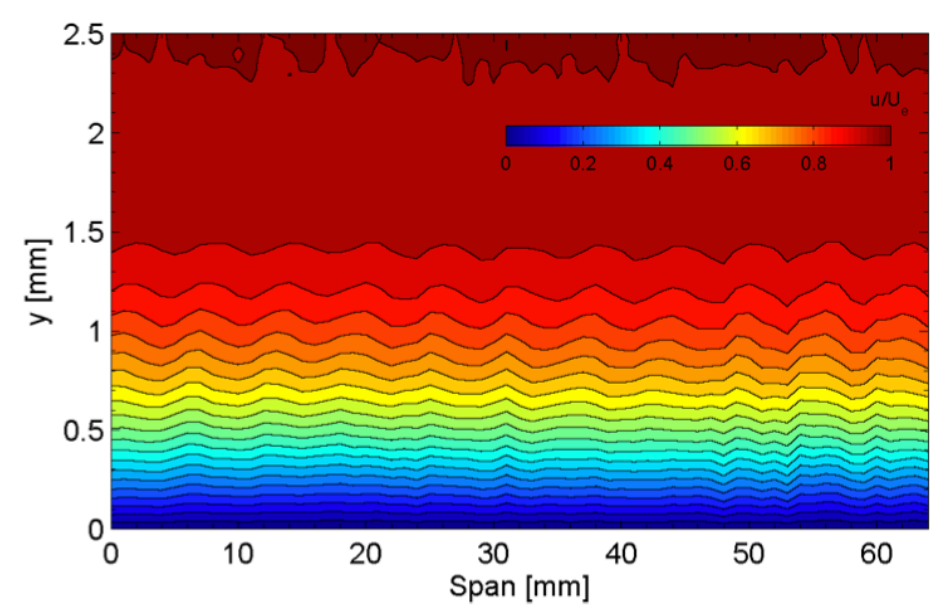

LES

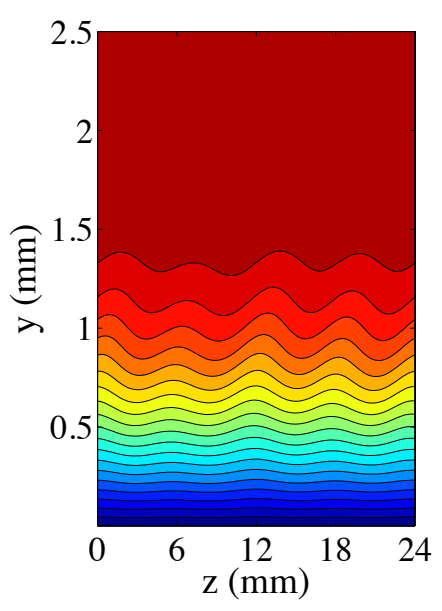

(a) 

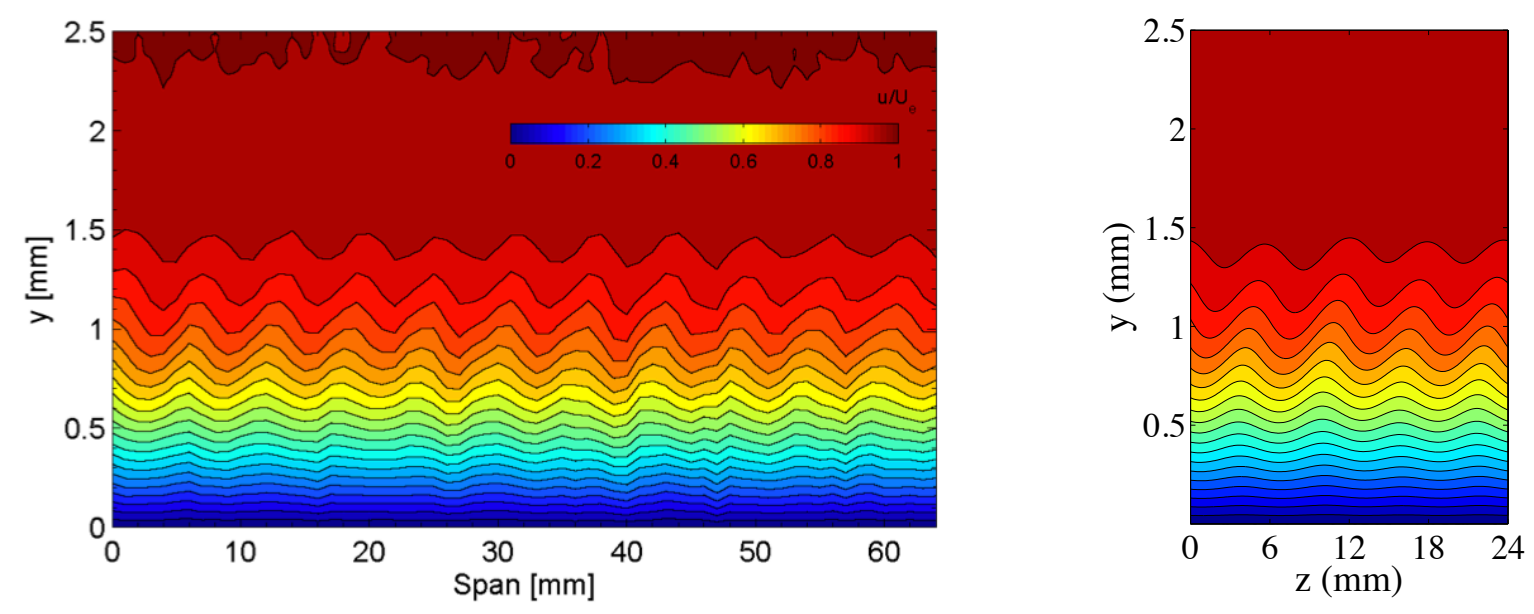

(b)
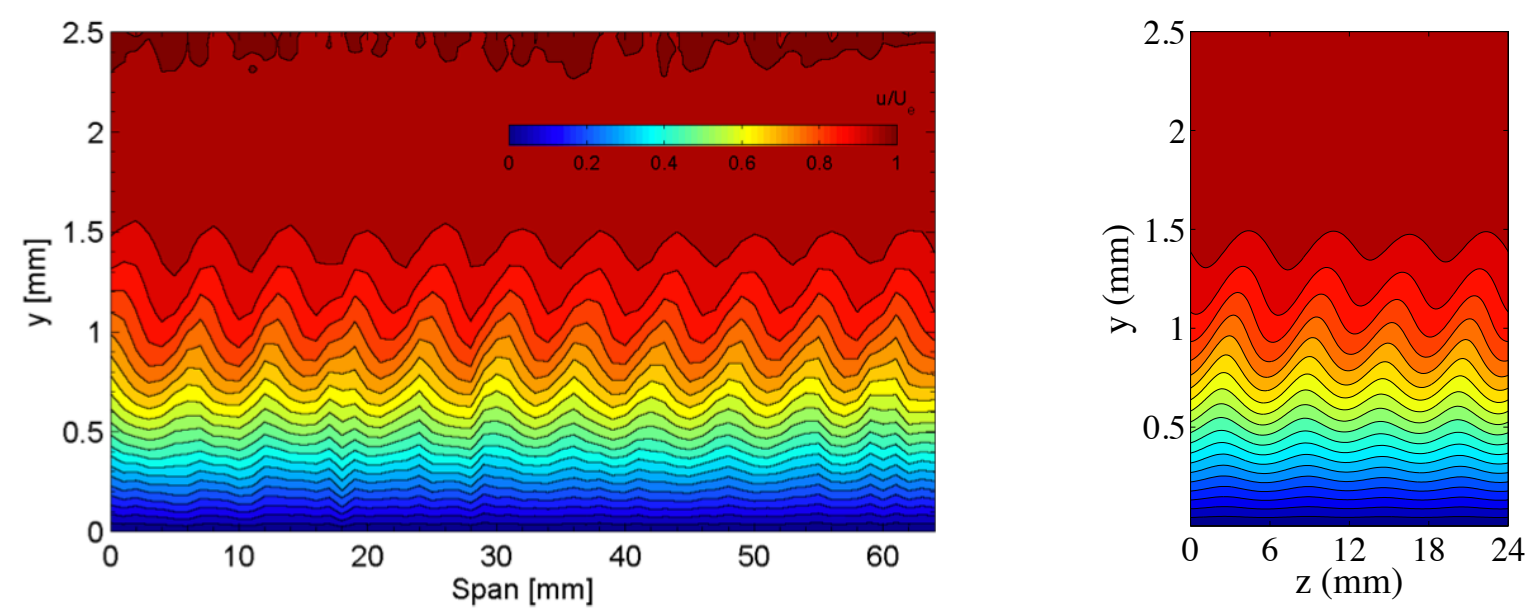

(c)

Figure 13: Contours of $u / U_{e}$ at $x / c=15 \%$ for 3 roughness heights, $\lambda=6 \mathrm{~mm}$ a) $k=14 \mu \mathrm{m}, b) \mathrm{k}=27 \mu \mathrm{m}, \mathrm{c}) \mathrm{k}=42 \mu \mathrm{m}$; experiment (left), simulation (right) 


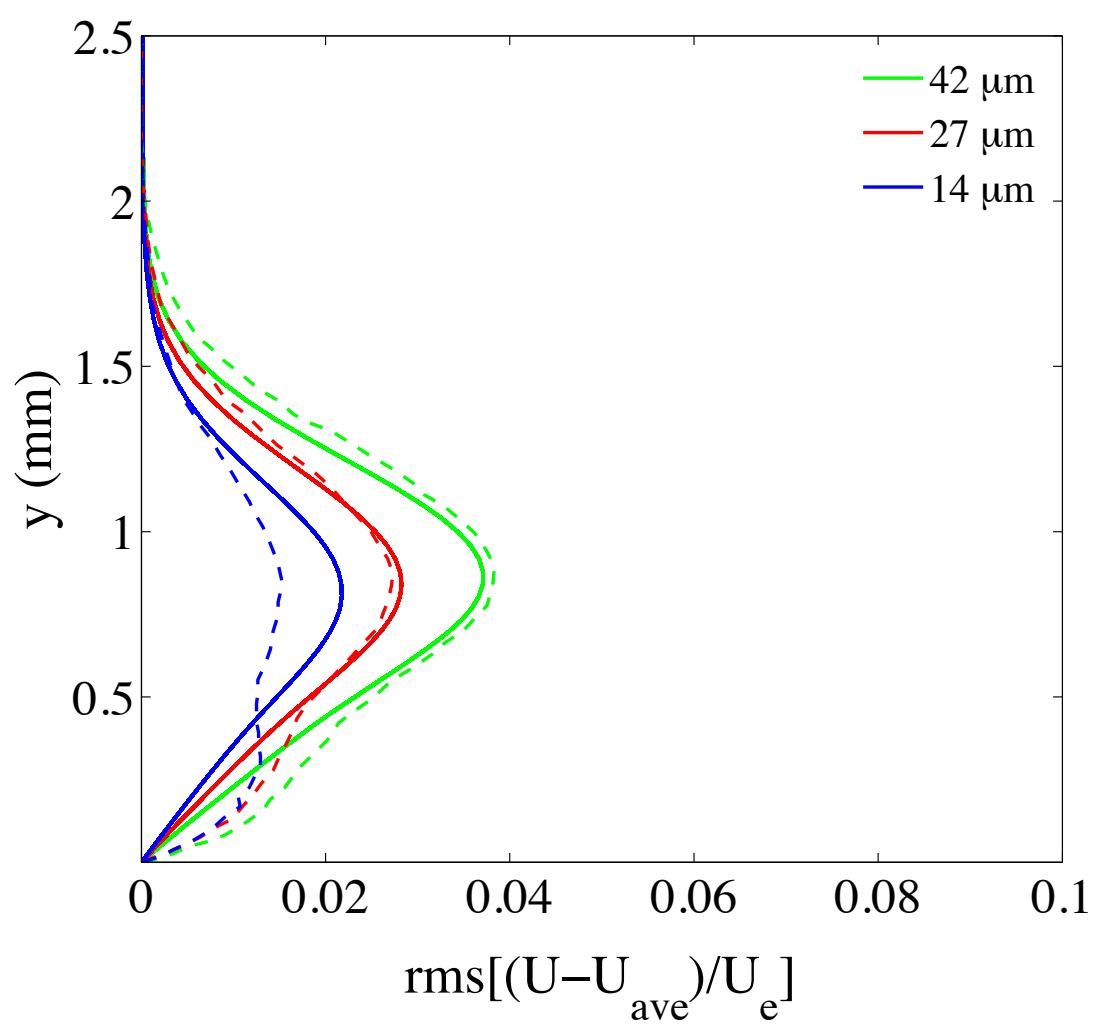

(a)

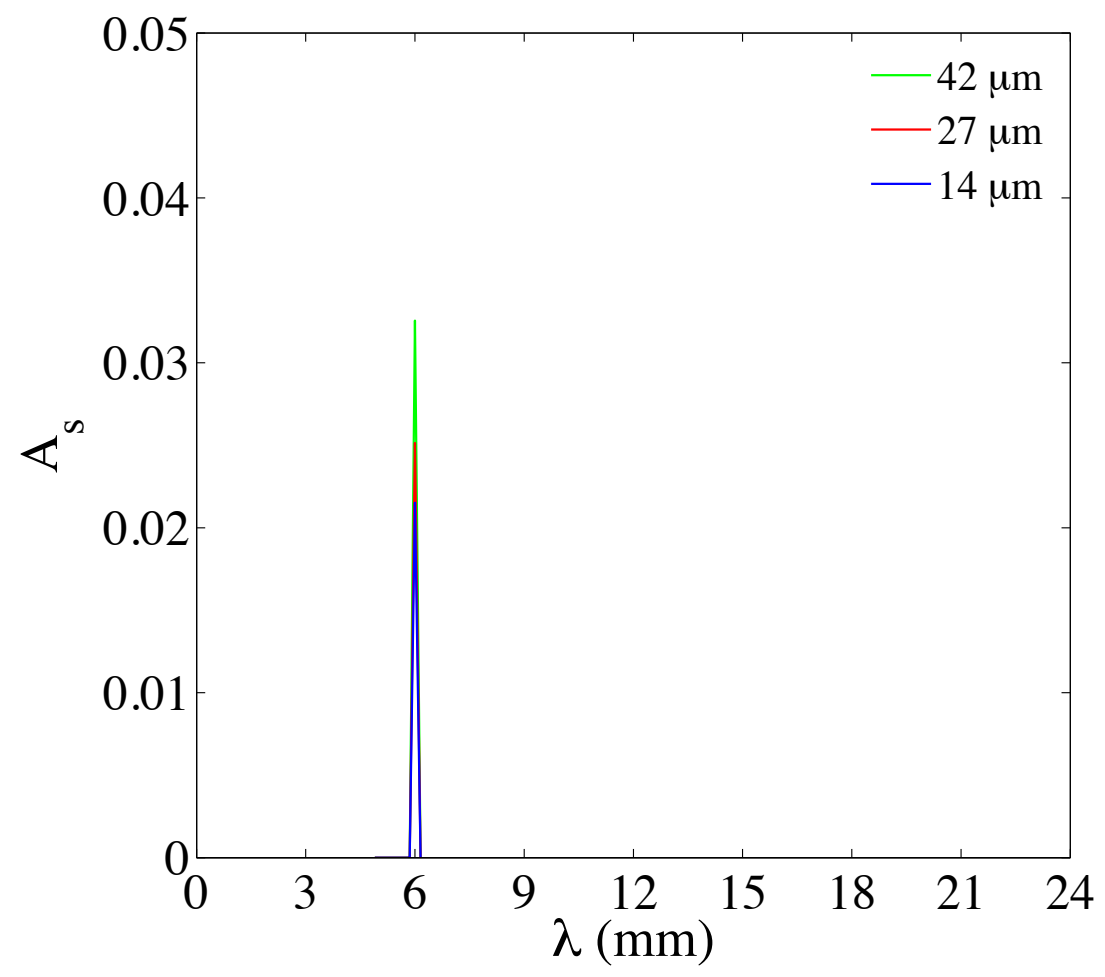

(b)

Figure 14: (a) Disturbance amplitude profile and (b) modal analysis for $\lambda=6 \mathrm{~mm}$ at $\mathrm{x} / \mathrm{c}=15 \%$ Solid lines - simulation; dashed lines - Expts. [15] 


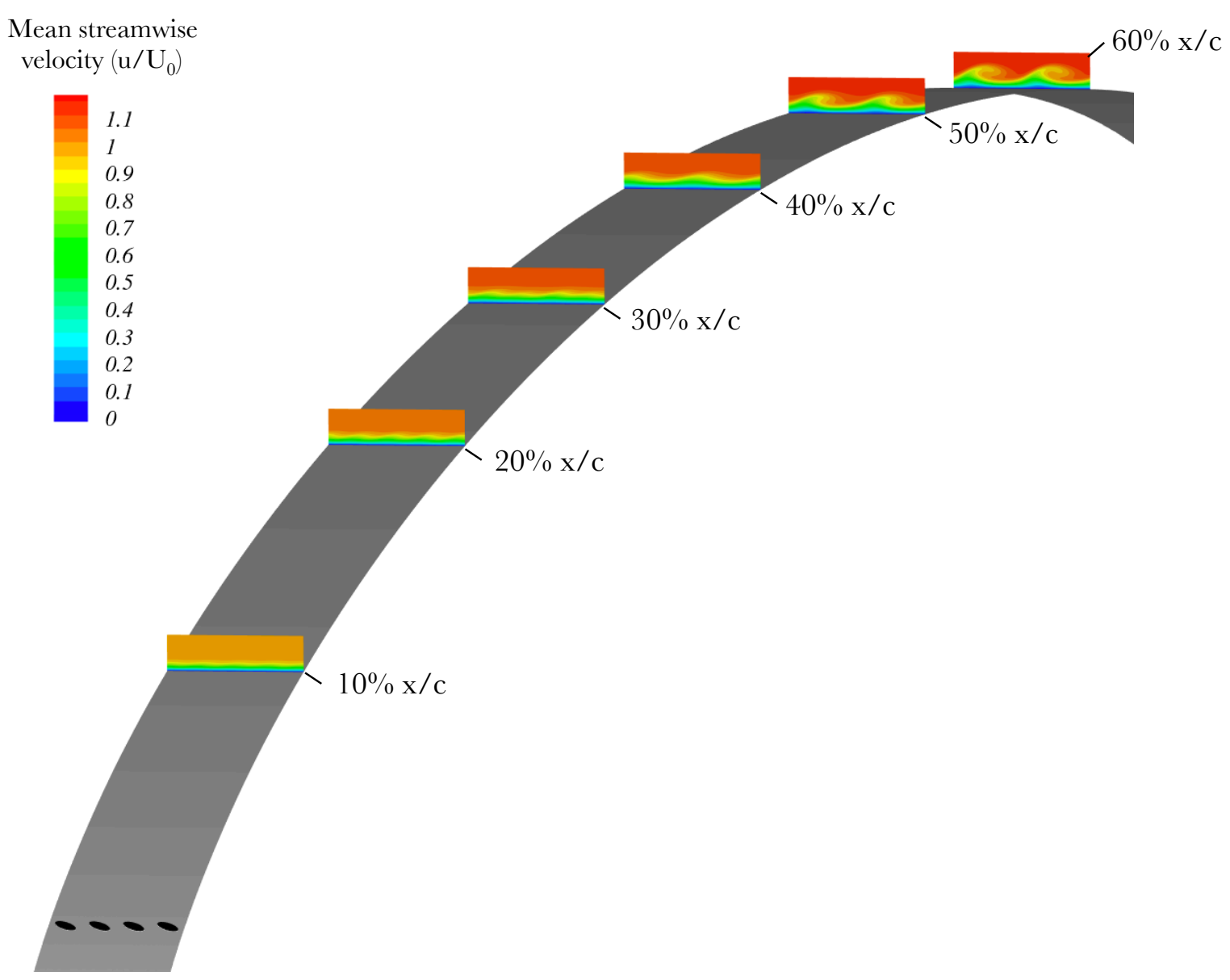

Figure 15: Axial velocity contours, $\lambda=6 \mathrm{~mm}, k=42 \mu \mathrm{m}$ 


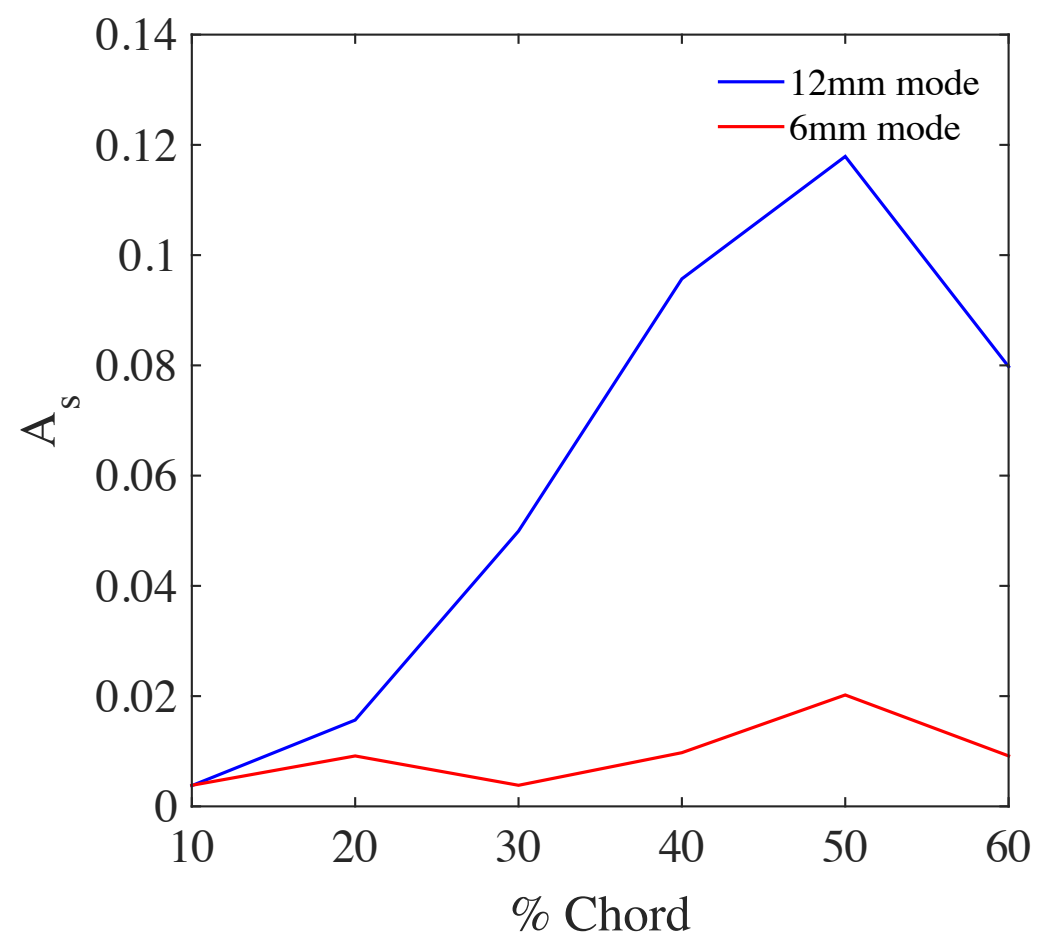

(a)

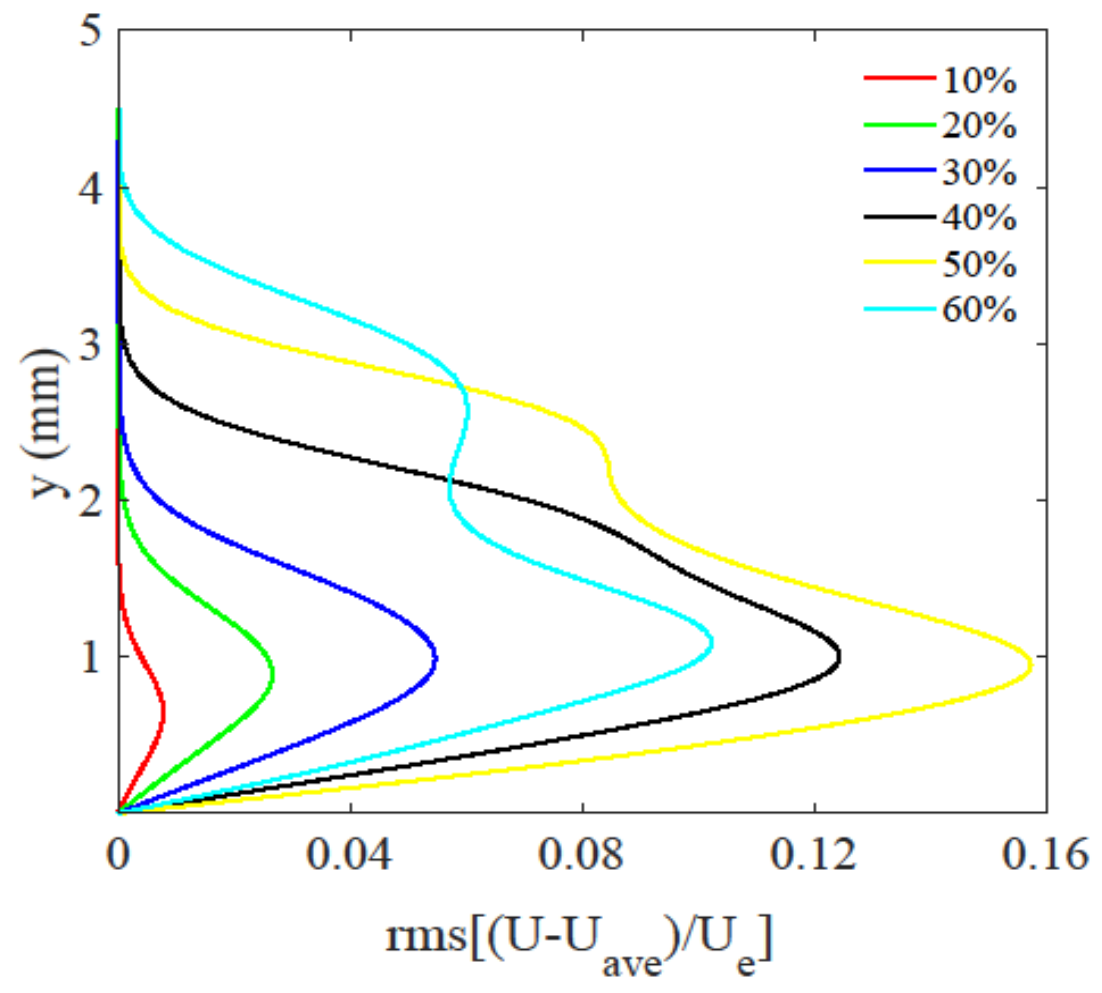

(b)

Figure 16: Evolution of (a) peak modal amplitudes and (b) rms disturbance mode shape $-k=12 \mu \mathrm{m}, \lambda=12 \mathrm{~mm}$ 


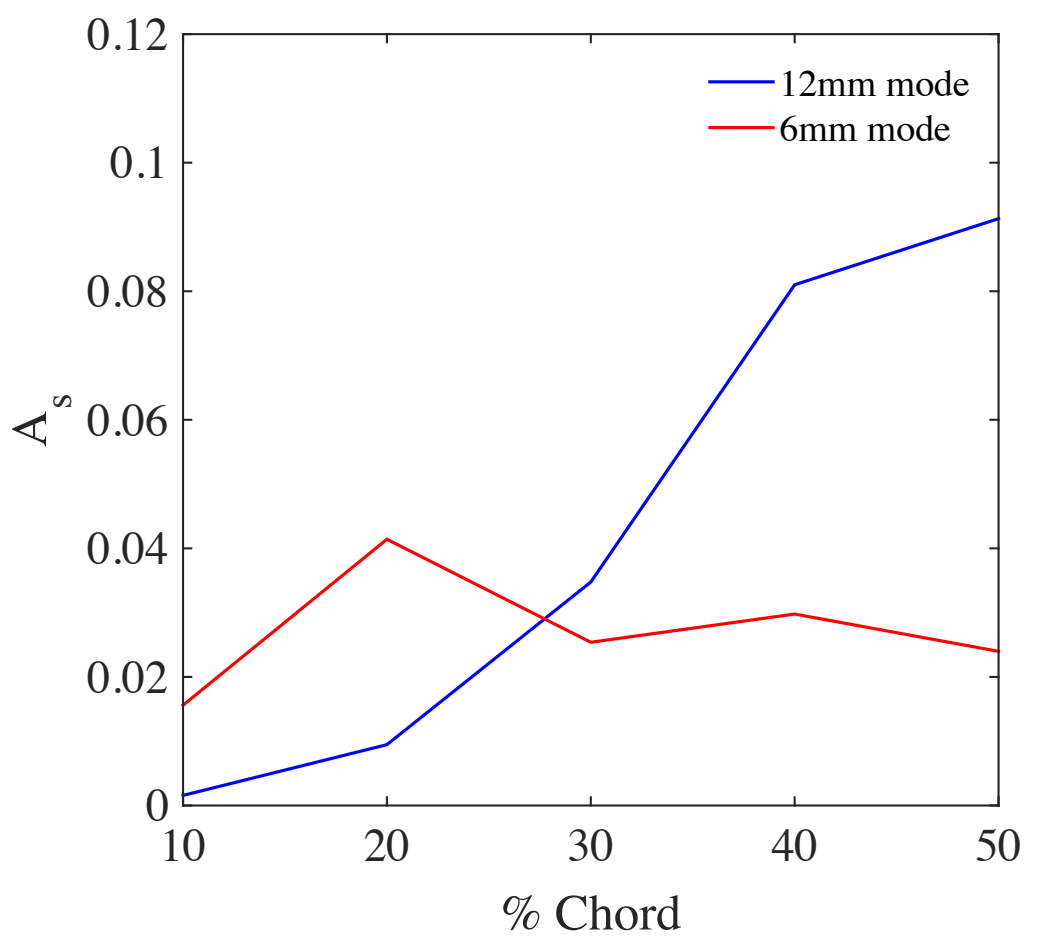

(a)

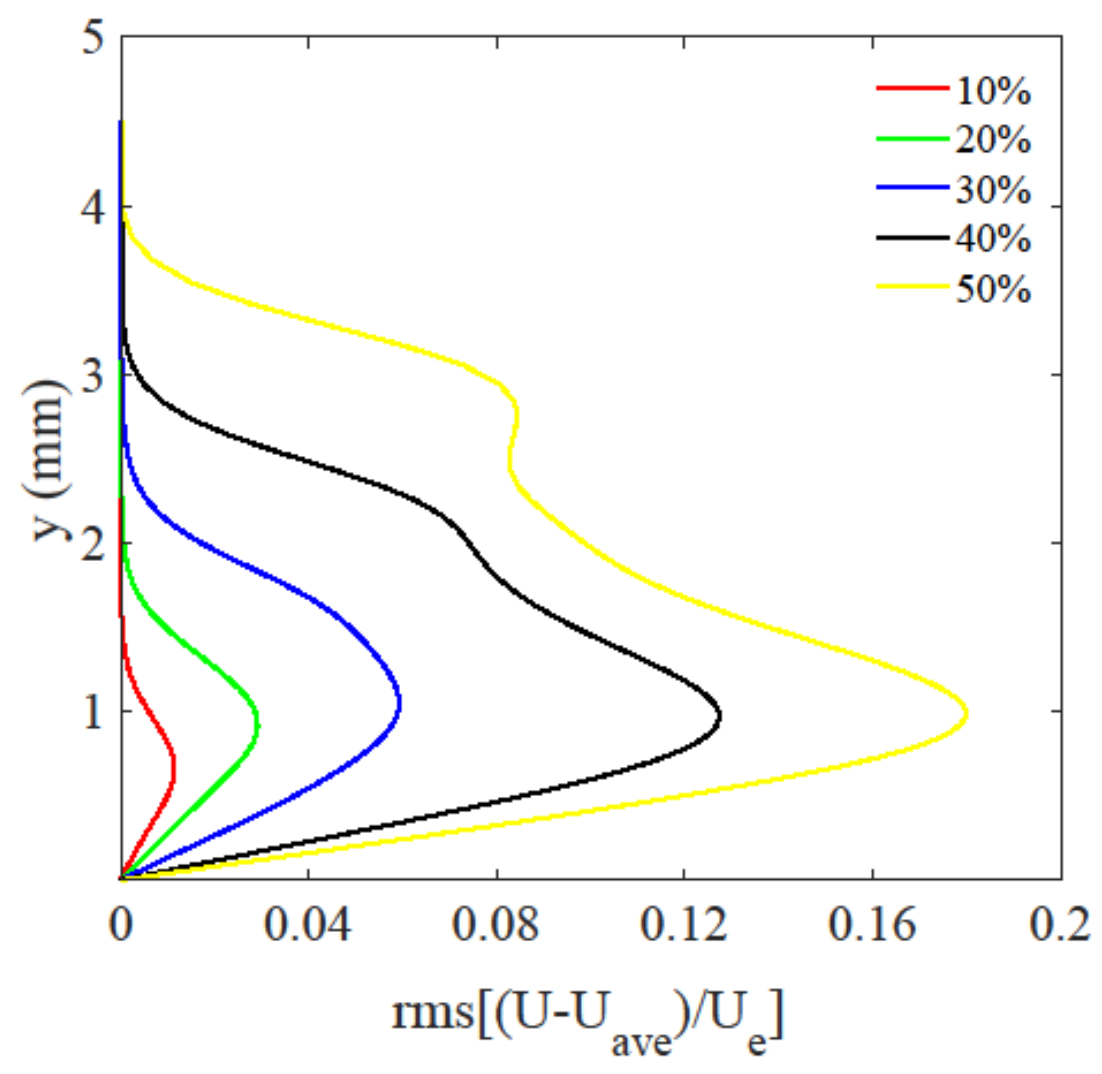

(b)

Figure 17: Evolution of (a) peak modal amplitudes and (b) rms disturbance mode shape - $k=14 \mu \mathrm{m}, \lambda=6 \mathrm{~mm}$ 


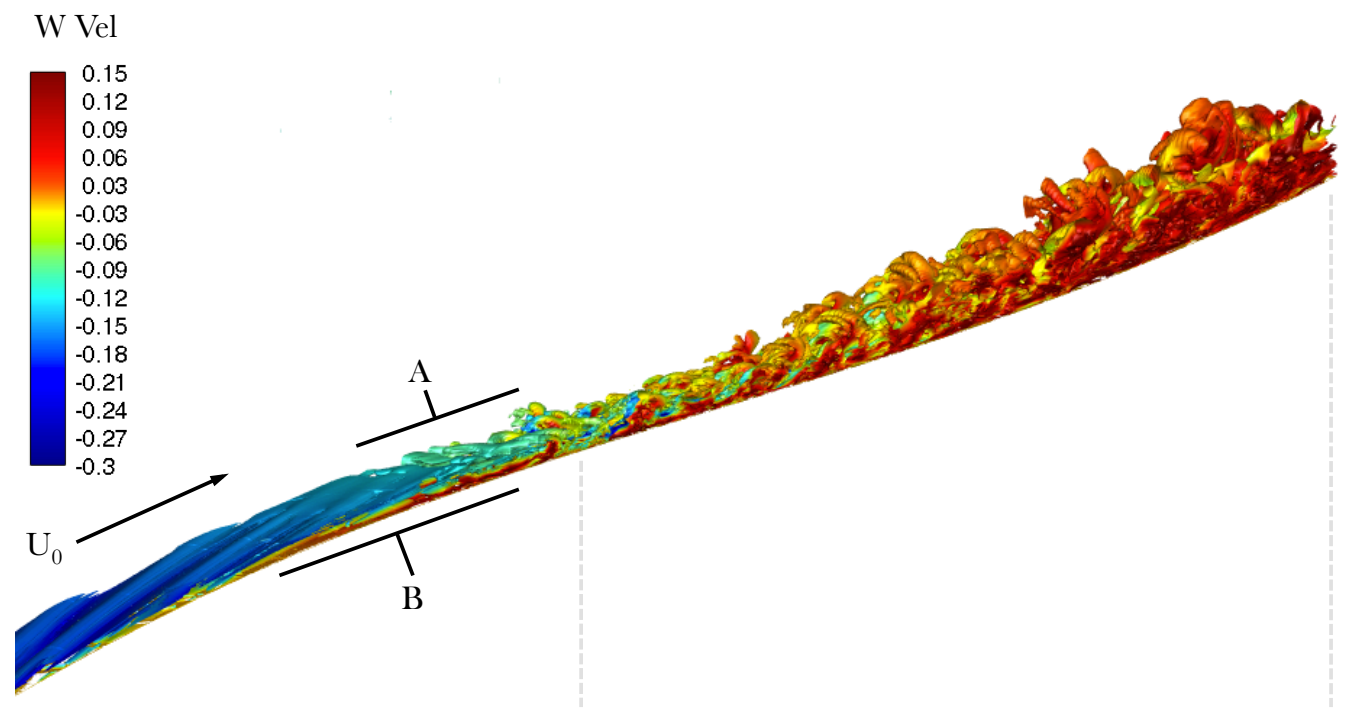

$80 \%$

$100 \%$

Figure 18: $\lambda_{2}$ iso-surface coloured by w velocity, case (i) (no FST) leading to breakdown into turbulence

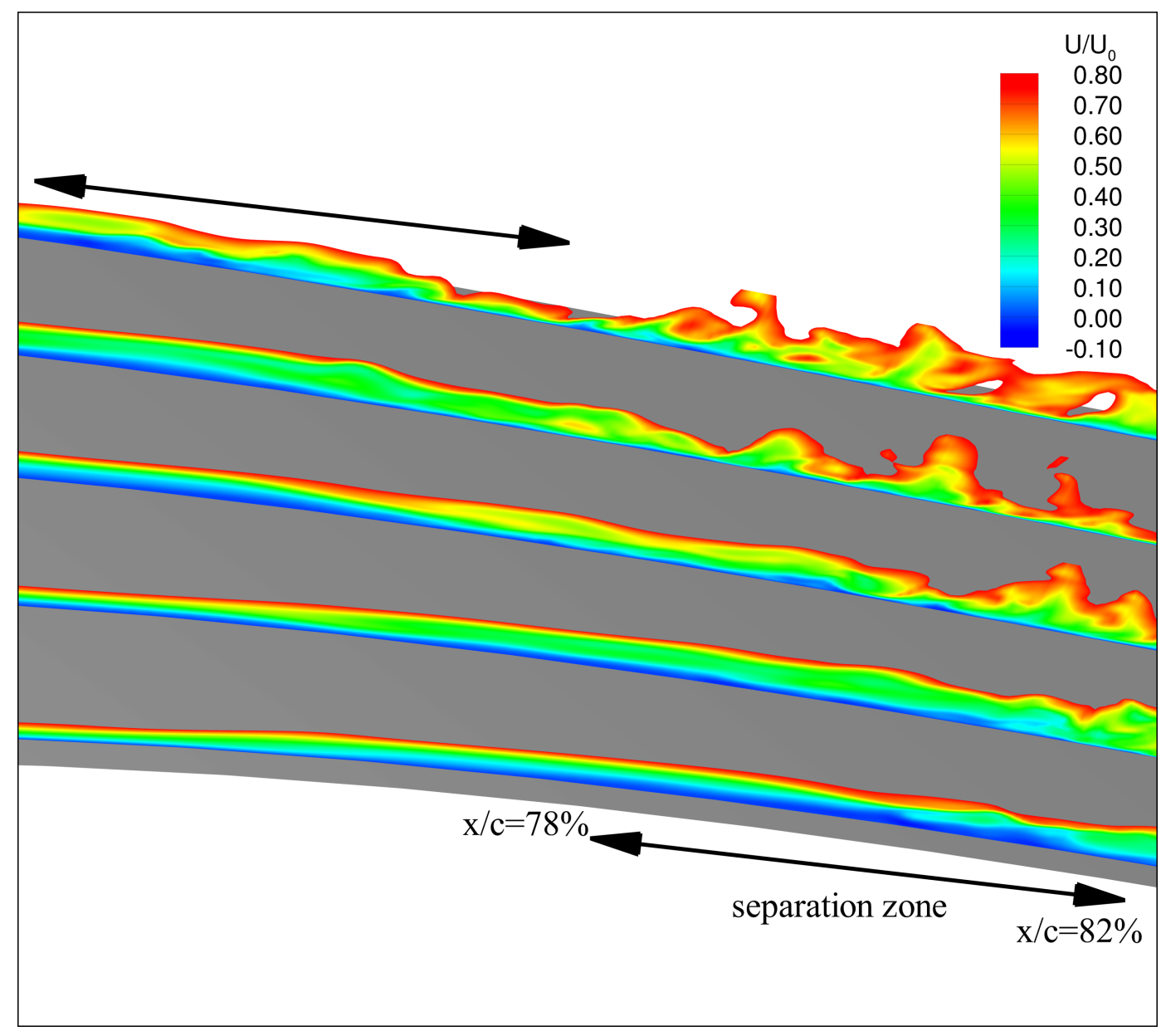

Figure 19: Zoomed in view of near wall flow - case (i) instantaneous axial velocity contours 


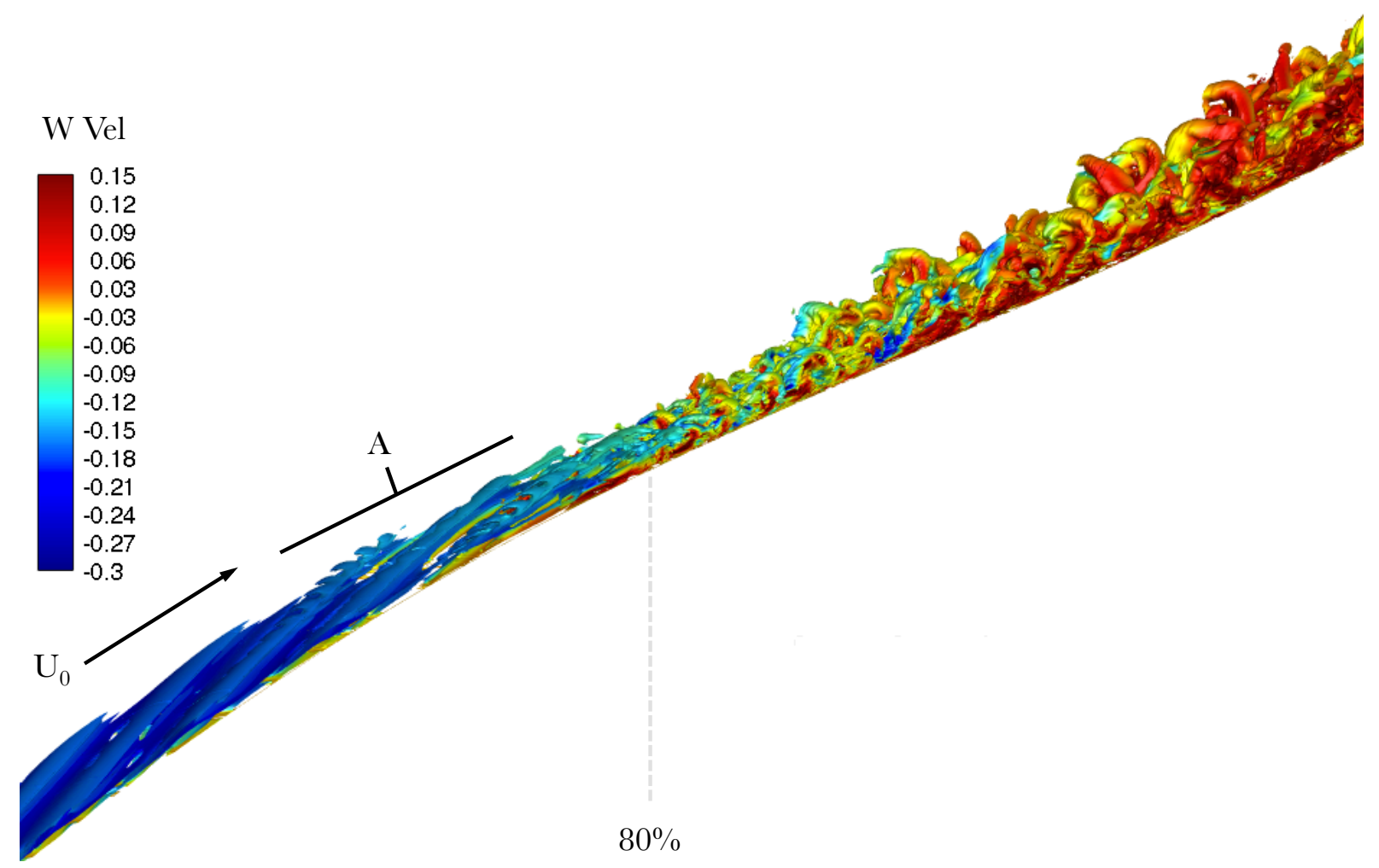

Figure 20: $\lambda_{2}$ iso-surface coloured by w velocity, case (ii) (FST added at $40 \%$ chord) leading to breakdown into turbulence 

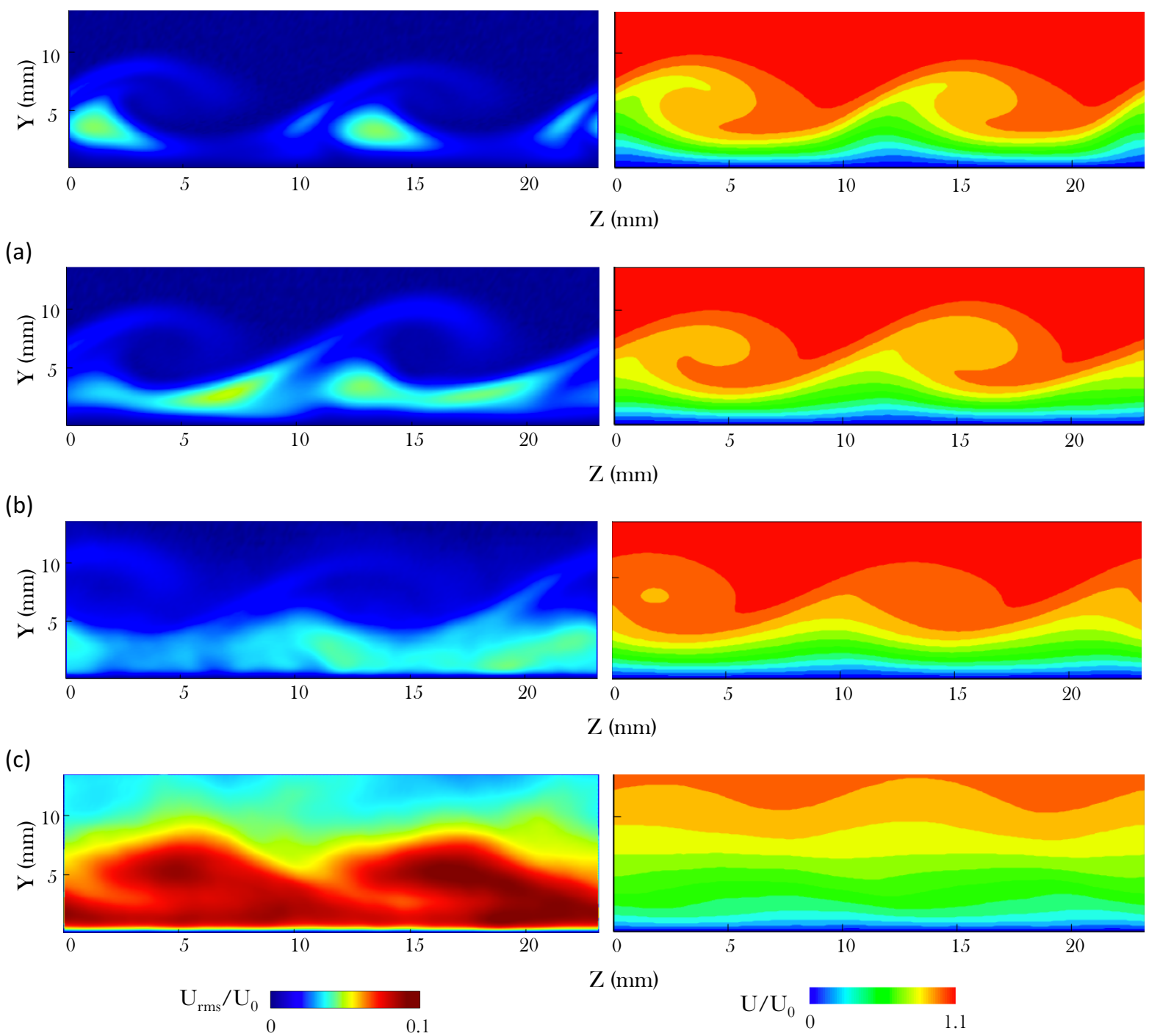

(d)

Figure 21: Left: Axial rms fluctuation contours, Right: mean axial velocity contours Case (ii) a) $x / c=50 \%$, b) $x / c=60 \%, c) x / c=70 \%$, d) $x / c=80 \%$ 


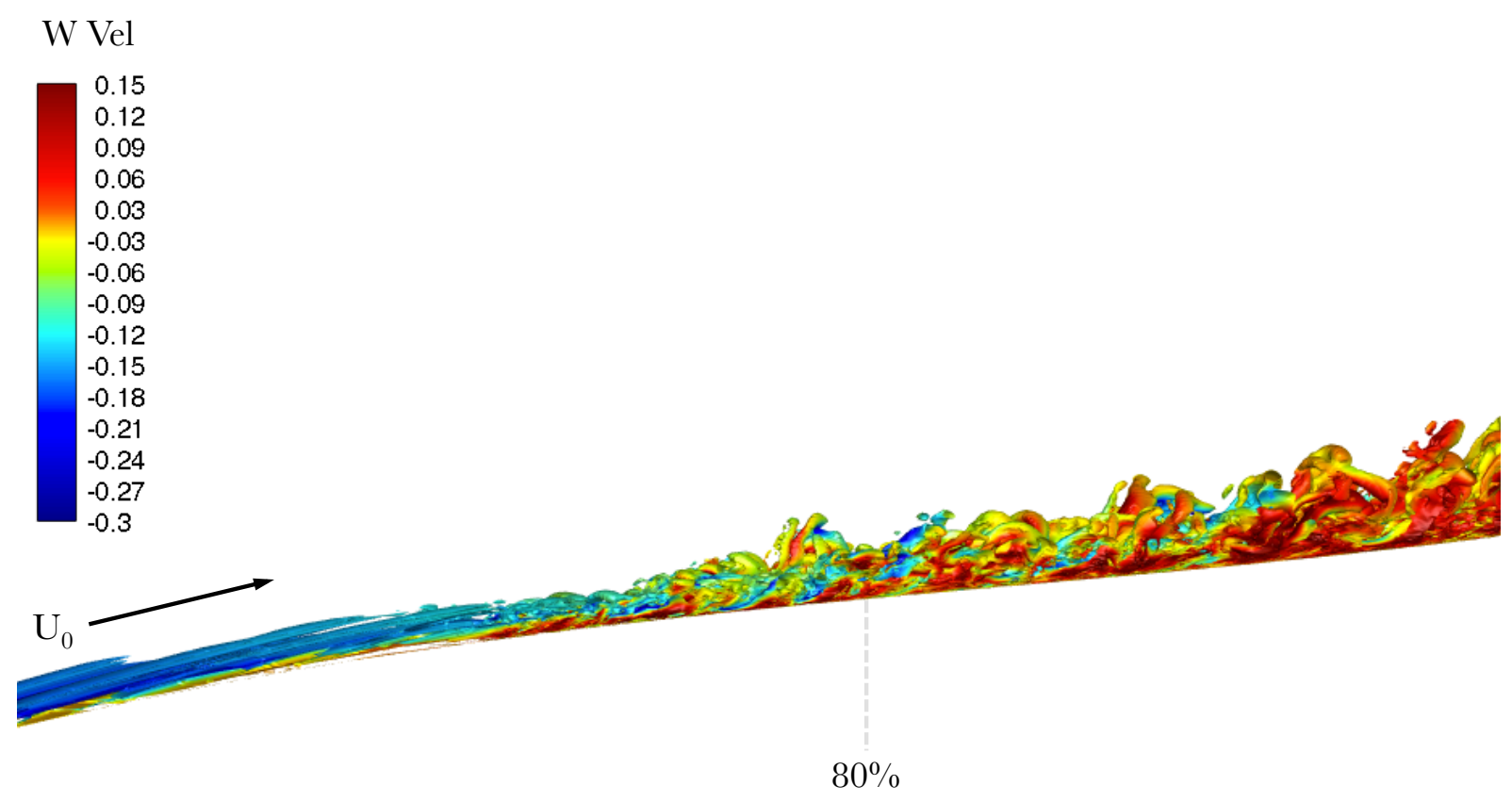

Figure 22: $\lambda_{2}$ iso-surface coloured by w velocity, case (iii) (FST added at $40 \%$ chord) leading to breakdown into turbulence 
No Freestream Turbulence $(\mathrm{k}=12 \mu \mathrm{m}, \lambda=12 \mathrm{~mm})$

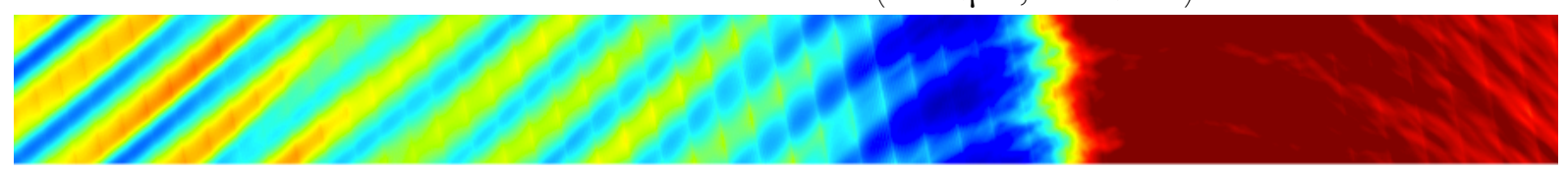

SEM Freestream Turbulence $(\mathrm{k}=12 \mu \mathrm{m}, \lambda=12 \mathrm{~mm})$

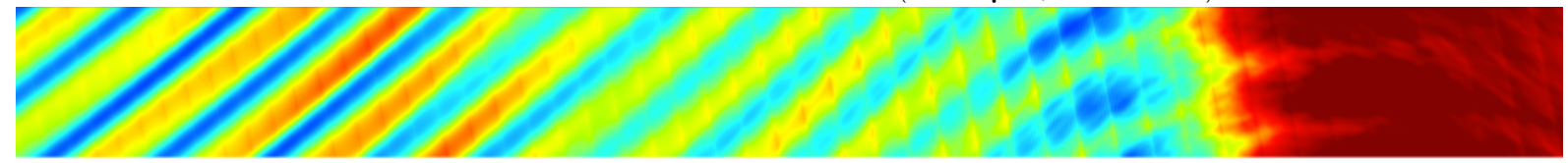

SEM Freestream Turbulence $(\mathrm{k}=14 \mu \mathrm{m}, \lambda=6 \mathrm{~mm})$

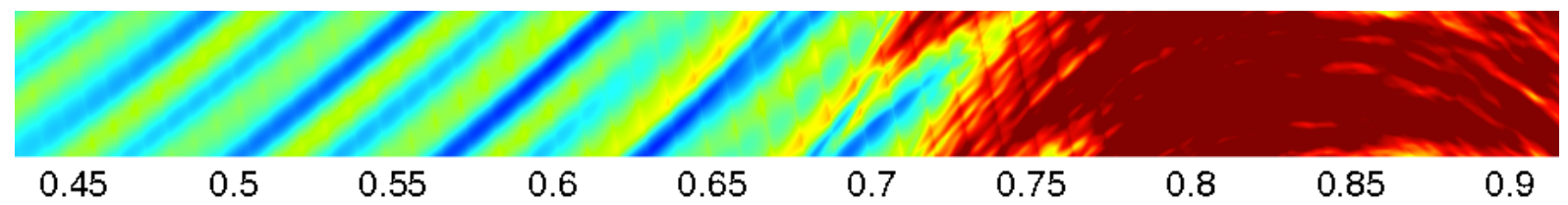

$\mathrm{x} / \mathrm{C}$

Skin Friction Coefficient $\left(\mathrm{C}_{\mathrm{f}}\right)$

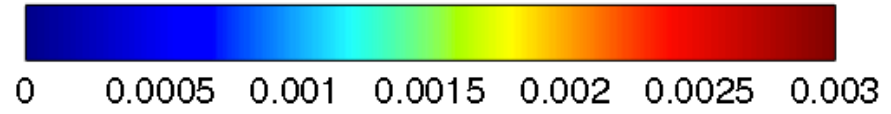

Figure 23: Contour plots of skin friction coefficient $\left(C_{f}\right)$ for 3 cases studied:

(i) $\mathrm{k}=12 \mu \mathrm{m}, \lambda=12 \mathrm{~mm}$, no freestream turbulence

(ii) $\mathrm{k}=12 \mu \mathrm{m}, \lambda=12 \mathrm{~mm}$, with freestream turbulence

(iii) $\mathrm{k}=14 \mu \mathrm{m}, \lambda=6 \mathrm{~mm}$, with freestream turbulence 